ARTICLE

\title{
Landscape of adenosine-to-inosine RNA recoding across human tissues
}

Orshay Gabay (10) ', Yoav Shoshan², Eli Kopel (1) 1, Udi Ben-Zvi1, Tomer D. Mann³, Noam Bressler², Roni Cohen-Fultheim (1) ${ }^{1}$, Amos A. Schaffer ${ }^{1}$, Shalom Hillel Roth ${ }^{1}$, Ziv Tzur ${ }^{1}$, Erez Y. Levanon (1) ${ }^{1,4 凶} \&$ Eli Eisenberg (10 ${ }^{2 凶}$

RNA editing by adenosine deaminases changes the information encoded in the mRNA from its genomic blueprint. Editing of protein-coding sequences can introduce novel, functionally distinct, protein isoforms and diversify the proteome. The functional importance of a few recoding sites has been appreciated for decades. However, systematic methods to uncover these sites perform poorly, and the full repertoire of recoding in human and other mammals is unknown. Here we present a new detection approach, and analyze 9125 GTEx RNA-seq samples, to produce a highly-accurate atlas of 1517 editing sites within the coding region and their editing levels across human tissues. Single-cell RNA-seq data shows protein recoding contributes to the variability across cell subpopulations. Most highly edited sites are evolutionary conserved in non-primate mammals, attesting for adaptation. This comprehensive set can facilitate understanding of the role of recoding in human physiology and diseases.

\footnotetext{
${ }^{1}$ The Mina and Everard Goodman Faculty of Life Sciences, Bar-Ilan University, Ramat Gan 5290002, Israel. ${ }^{2}$ Raymond and Beverly Sackler School of Physics and Astronomy and Sagol School of Neuroscience, Tel Aviv University, Tel Aviv 6997801, Israel. ${ }^{3}$ Tel Aviv Sourasky Medical Center and Sackler school of medicine, Tel Aviv University, Tel Aviv, Israel. ${ }^{4}$ The Institute of Nanotechnology and Advanced Materials, Bar-Ilan University, Ramat Gan 5290002, Israel.




A denosine-to-inosine (A-to-I) RNA editing, catalyzed by the ADAR (adenosine deaminases acting on RNA) family of enzymes ${ }^{1-4}$, is the most common form of RNA editing among animals ${ }^{5}$. As the translation machinery largely interprets inosines as guanosines ${ }^{6}$ editing within the coding sequence (CDS) may lead to amino-acid substitution ("recoding") and diversify the proteome. Recoding of GRIA2 (Glutamate Ionotropic Receptor AMPA Type Subunit 2) Q/R site is essential in mammals ${ }^{7}$, and recoding of a few additional well-studied targets has been shown to be functionally important. Notable examples are the antizyme inhibitor 1 (AZIN1) ADAR1-dependent recoding that promotes cell proliferation and contributes to cancer progression ${ }^{8}$, recoding of NEIL1 (Nei Like DNA Glycosylase 1) that results in a 30 -fold reduction in the thymine glycol cleavage rate when acting on duplex $\mathrm{DNA}^{9}$, and FLNA (Filamin A) recoding which regulates vascular contraction and diastolic blood pressure ${ }^{10}$. Despite the potential importance demonstrated by these few examples, the full repertoire of human recoding sites is not known, and the scope of its functionality is still unclear.

As part of standard sequencing protocols, inosines in the RNA are reverse-transcribed into guanosines in the cDNA. Thus, aligning the sequencing reads to the reference genome, A-to-I editing is manifested as A-to-G genome-read mismatches. Typically, recoding activity is dwarfed by A-to-I editing events at millions of sites within non-coding regions ${ }^{5}$, and is therefore much more difficult to detect (Fig. 1a). As a result, systematic analyses of mismatches yielded high-specificity identification of sites in human ${ }^{5,11-17}$, virtually all of them within Alu repeats, but performed poorly in coding regions. The relatively small number of recoding sites ${ }^{14,18}$ is overshadowed by additional sources of discrepancies between the reads and the reference genome. First, genomic variability between the reference genome and the sampled individuals translates into mismatches between the reference genome and the RNA sequenced from these individuals. Over 500 million human single-nucleotide-polymorphisms (SNPs) have been identified so far, of which almost all are rare. A typical individual genome includes tens of thousands of rare polymorphisms ${ }^{19}$. Matching DNA-seq data could be used to filter out these sites per sample, but it is usually not available ${ }^{20}$. Furthermore, even when available, somatic genomic mutations may result in mismatches misidentified as editing events. Second, systematic misalignment of RNA-seq reads to the wrong (but homologous) genomic locus could lead to an apparently consistent mismatch, to be misidentified as an editing event ${ }^{21-23}$. These are common in the CDS as many proteins has closelyrelated paralogs, polymorphic duplications, and processed pseudogenes. These mapping problems are enhanced by splicing (especially when genomic polymorphisms reside in proximity to canonical splicing junctions) ${ }^{21,22}$. Moreover, each individual genome harbors several megabases of non-canonical segments ${ }^{24,25}$, which commonly include unique duplications and processed pseudogenes. Chimeric sequences and spontaneous deamination contribute to the observed mismatches as well. Finally, technical sequencing errors are customarily cleaned using the quality score. However, the quality score seems to underestimate the error rate in specific locations such as homopolymeric sequences ${ }^{26}$ or reads' ends, as well as the vicinity of mispriming events by the random hexamer primers ${ }^{27}$. Thus, to date, the low specificity and sensitivity of RNA-editing site detection within the coding sequence ${ }^{28}$ hinder global functional analyses and evolutionary studies of recoding in human.

Here we present a novel de-novo RNA editing detection approach, dedicated and optimized for the coding region. Analyzing 9125 human RNA-seq samples from various tissues (GTEx data $)^{29}$, we produce a set of 1517 A-to-I RNA editing sites within protein-coding regions, with an estimated false positive rate of only $8 \%$. Surprisingly, recoding is found in most tissues at similar levels and is not enriched specifically in the brain. There is a wide range of editing efficiencies, and a few target sites account for the majority of recoding activity. Yet, the variation of the editing level per-site across individuals is generally low, implying regulation. Most strongly-edited sites are conserved across mammals, and thus recoding as a whole seems to be under positive selection. Unexpectedly, we find a number of large clusters of recoding sites, which could lead to an extensive combinatorial diversity of the affected transcripts. Analyzing mass-spectrometry data, we provide evidence that editing at these sites results in modified proteins. Finally, we show many recoding sites to be differentially edited in cancer and pneumonia, suggesting possible etiological relevance.

\section{Results}

A new pipeline for detecting editing in the coding region. Previous searches and existing detection tools (e.g., SPRINT ${ }^{30}$, REDItools $^{31}$, or RNAEditor ${ }^{32}$ ) for editing in human coding regions have resulted in low sensitivity and precision ${ }^{27}$ (Fig. $1 \mathrm{~b}$ and Supplementary Fig. 1), due to the abovementioned difficulties. Precision has been much improved using a more stringent alignment scheme ${ }^{33}$. However, this approach is highly timeconsuming and is not applicable for large datasets such as GTEx. Here we present a detection pipeline designed specifically to overcome these issues (Fig. 1c and "Methods"). Briefly, we focused on non-repetitive protein-coding regions, excluding, in particular, the editing-rich Alu exons, and analyzed 9125 RNAseq normal human samples from the GTEx project (548 donors, 47 tissue types; Supplementary Data 1$)^{34}$. We applied strict alignment procedures, discarding mismatches that are likely to be explained by systematic alignment or sequencing errors (Supplementary Fig. 2), and a statistical model that integrates the cumulative profile of mismatches found for all donors of a given tissue type into a single score. Using this score, we filtered out mismatches found in a small number of donors, possibly due to rare SNPs and duplications. We masked genomic loci where multiple closely-located mismatches of different types were observed, as they are suspected to result from misalignments, and used whole-exome-sequencing (WES) data to discard mismatches observed in genomic reads and further suppress false-positive detections. The breadth and depth of GTEx data allow for a reliable and consistent detection of low-level editing, as well as detection in tissues in which editing has not been studied so far. However, sites that are rarely edited $(<1 \%$ editing level in all tissue types) were discarded, as they are harder to detected reliably and less likely to be functionally relevant.

This pipeline resulted in a reliable set of 1517 human CDS Ato-I RNA sites (Fig. 2a and Supplementary Data 2), showing the familiar $5^{\prime}$ and $3^{\prime}$ neighbor-sequence preferences of mammalian ADARs $^{35}$ (Fig. 2b). The large number of T-to-C sites (1006) also exhibit the familiar (reverse-complement) motif (Fig. 2c), suggesting that they are mostly due to A-to-I editing of RNA molecules transcribed from the antisense strand, complementary to the annotated coding RefSeq exons (Fig. 2d). To verify that, we analyzed a strand-specific RNA-seq dataset ${ }^{36}$, for which one can separate the reads based on their genomic strand of origin. As expected, the T-to-C signal observed in GTEx non-stranded data does not show up in reads originating from the coding strand and is fully accounted for by A-to-G editing of reads transcribed from the non-coding strand (Fig. 2e). Based on these results, we estimate that only $\sim 24$ of the $1006 \mathrm{~T}$-to-C sites observed are falsepositive detections ("Methods"). Note that the editing levels found at the detected T-to-C sites using stranded data are $\sim 3$-fold higher than the A-to-G sites, due to a lower detection power in 
a

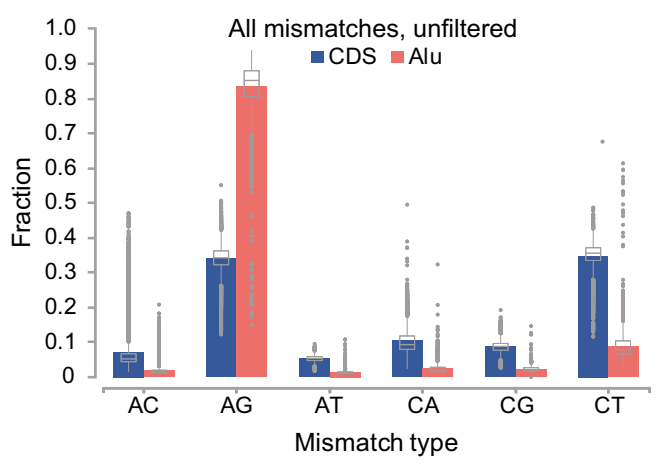

b

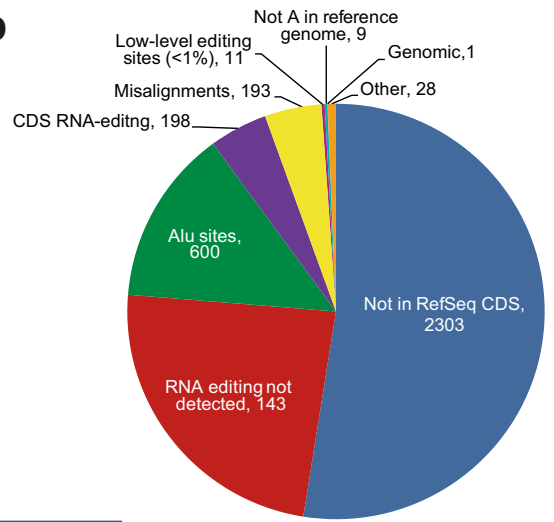

C

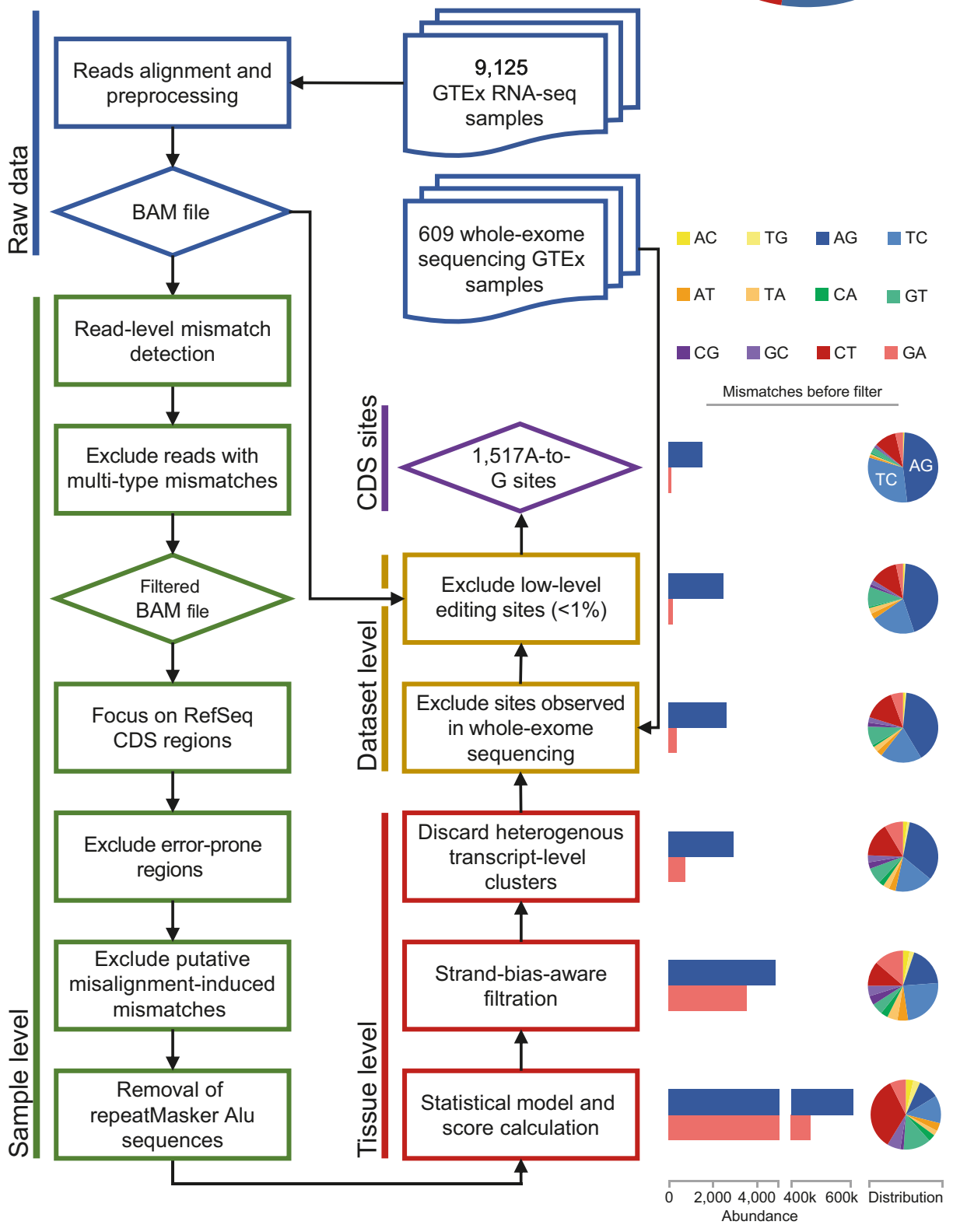

the antisense strand. Expression of this strand is typically lower compared to the protein-coding strand (see Supplementary Data 3), and thus the editing signal is masked by the many unedited reads coming from the sense strand. Accordingly, the sites that are detected under these conditions are typically more strongly edited, and the editing levels reported for these sites based on non-stranded data are underestimated. Some of the C-
to-T sites detected (mostly in blood samples, where APOBEC3A is highly expressed) may reflect DNA or RNA editing by members of the AID/APOBEC family of deaminases. Indeed, one of the detected sites is the only known $\mathrm{C}$-to- $\mathrm{U}$ recoding site in


abundant mismatch type, and estimated the false positive rate to be $8 \%$ (115 G-to-A sites). 
Fig. 1 A high-precision CDS-focused RNA editing detection pipeline. a Relative abundance of all six types of RNA-DNA mismatches (strand-insensitive, i.e., A-to-C includes also T-to-G, etc.) following an alignment of 9125 GTEx RNA-seq samples to the reference human genome. All mismatch events are included, with no filtering. Multiple mismatches to the same genomic position are counted as separate events. Enrichment of A-to-G mismatches, presumably due to A-to-I RNA editing events is readily detectable within Alu elements. However, no such enrichment is observed in Alu-free CDS, where the editing signal is dwarfed by the noise. Colored bars and box-and-whisker plots represent the mean and the full distribution, respectively, of the relative abundances. See Supplementary Data 1 for the number of biologically independent samples per tissue. $\mathbf{b}$ Classification of all A-to-I sites annotated in REDIportal ${ }^{12}$ database as CDS. Merely 198 out of 4386 sites (4.5\%) were detected by our pipeline as reliable CDS RNA editing sites while the majority of the sites were excluded from our analysis due to the reasons indicated in the panel. c A flowchart summarizing the main steps of our CDS RNA editing detection pipeline. Briefly, 9125 GTEx RNA-seq samples from various donors and tissues were aligned to the reference genome and DNA-RNA mismatches were detected and filtered within each sample separately. Results were aggregated for each tissue type for further filtration steps. Finally, the resulting candidate sites were filtered using global dataset criteria to yield a final 1517 reliable CDS A-to-I RNA editing sites (see "Methods" for details). Rightmost panel shows the mismatches abundance and distribution before each of the final filtering steps, demonstrating the increase in signal-to-noise ratio per step. Source data are provided as a Source Data file.

ADAR enzymes bind to dsRNA secondary structures ${ }^{39}$. Accordingly, the predicted editing sites tend to be part of putative intra-molecular dsRNA structures (Fig. $2 \mathrm{f}$ and "Methods"). To test whether editing of these sites translates into novel protein isoforms, we have analyzed 311 human mass-spectrometry proteomic samples taken from the PRIDE database ${ }^{40}$ (Supplementary Data 4). Shotgun proteomics results in partial coverage of the tryptic peptides ${ }^{41}$, and thus only 138 of the sites are covered by peptides, regardless of their editing state. Of these, peptides supporting the edited version of the transcripts were observed for 35 sites (25\%) ("Methods", Fig. 2g and Supplementary Data 5). Reassuringly, edited peptides were observed for $65 \%$ $(11 / 17)$ of the covered sites predicted to be appreciably edited ( $>5 \%$ editing in RNA-seq data).

The sensitivity of our pipeline is demonstrated by recovering all 38 previously published mammalian conserved sites ${ }^{42}$, except for one of the IGFBP7 (Insulin Like Growth Factor Binding Protein 7) sites $^{43}$ that resides adjacent to a homopolymeric sequence and was, thus, filtered out. However, the overlap of our list with previously published sets of human CDS sites ${ }^{12}$ is rather low (Supplementary Fig. 3), which we attribute to low specificity and sensitivity of previous approaches. Indeed, for most of the previously published sites we can pinpoint the reason for their misidentification (Fig. 1b).

Tissue-specificity of CDS editing sites. Editing in the coding region is not specific to brain regions. The number of sites discovered per tissue is similar for arteries, lung, and brain tissues (Fig. 3a). Furthermore, quantification across tissues of the global editing levels in the coding sequence (the RNA editing index $^{33,44,45}$, a weighted average over all sites; Methods) shows (Fig. $3 \mathrm{~b}$ ) that the arteries ${ }^{10}$, colon, and esophagus are edited to a much higher extent than the brain. However, most of the recoding signal in these tissues is due to a small number of targets (mainly the highly-expressed and highly-edited FLNA and IGFPB7 ${ }^{43}$ ) whereas editing in the brain is much more diverse and affects a larger number of targeted genes and sites (Fig. 3a and Supplementary Fig. 4). Interestingly, distinct editing profiles are found for different tissues (e.g., blood, testis, heart and muscle) (Fig. 3c). Sites are not enriched or depleted in annotated protein domains compared to other coding regions (incidence rates $1.6 \times 10^{-4}$ and $1.8 \times 10^{-4}$, respectively; Fisher's exact test $p=0.06$, not significant). Note that the distribution of editing levels is heavily skewed towards weakly-edited sites (Fig. 3d), and thus the number of sites declared as being edited depends strongly on the editing level cutoff.

Analyzing single-cell RNA-seq data (Methods), we find editing levels to vary dramatically between different cell populations within the same tissue ${ }^{46}$ (Supplementary Data 6, 7). Remarkably, the variation of the editing pattern across cell populations is complex, and cannot be explained by a simple regulation mechanism. For example, editing in the brain is significantly higher for endothelial cells compared to excitatory neurons for several sites (CCNI (Cyclin I), COPA (COPI Coat Complex Subunit Alpha), and AZIN1), but lower at the TMEM63B (Transmembrane Protein 63B) site. Similarly, for many sites (GRIA2, GRIA3, TMEM63B, CYFIP2 (Cytoplasmic FMR1 Interacting Protein 2), and KCNA1 (Potassium Voltage-Gated Channel Subfamily A Member 1)) editing is significantly higher for excitatory neurons compared to oligodendrocyte precursor cells in the brain, but lower at the COPA site. We note that excitatory and inhibitory neurons exhibit markedly different editing levels at two key sites known to regulate neural activity, GRIA2 RG site (editing level 92\% for excitatory neurons, compared to $61 \%$ in inhibitory neurons) and KCNA1 (69\% vs. $10 \%$ ). Interestingly, ADAR3 (also known as ADARB2), known to regulate RNA editing levels ${ }^{14,47}$, was previously implicated as one of the few genes differentiating between subpopulations of inhibitory neurons ${ }^{48}$.

Validation of results in additional datasets. In order to further validate our A-to-G results, and further support our conclusion that the large number of T-to-C sites result from A-to-I editing of RNA molecules transcribed from the antisense strand we have analyzed three additional strand-specific RNA-seq datasets (see "Methods"), together with the previously mentioned dataset ${ }^{36}$. Looking at the coding strand, we find that 1375 of the 1517 A-to$G$ sites were covered in at least one of the four datasets $(>100$ reads). Of these, 948 were found to be edited in at least one dataset (FDR corrected binomial $p$-value $<0.05$ for the pooled data, per dataset). Note that sites that did not show evidence of editing could be edited in other tissues (not present in this validation study) or missed due to a low editing level and insufficient coverage. For the 1006 T-to- $C$ sites, 885 were covered but only 40 (4.5\%) of them have passed the binomial test with $p<0.05$. Looking at the opposite strand, only 76 of the 1517 A-to-G sites were covered in at least one of the four datasets, and only 5 of them (6.6\%) have passed the binomial test with $p<0.05$, while $582 / 1006$ T-to-C sites were covered, and 545 of them were found to be edited. Taken together, these results demonstrate the accuracy of our set of editing sites, and the proposed explanation for the T-to-C mismatched observed in non-stranded data.

ADAR-specificity of CDS editing sites. ADAR2 (also known as ADARB1) is the main contributor to editing in the coding sequence. Quantifying editing at our sites upon ADARs overexpression in HEK 293 cell lines $^{14}$, we were able to classify 24 sites as ADAR1-dependent, 179 sites as ADAR2-dependent, and 24 sites were shown to be edited by both (Fig. $3 \mathrm{e}$ and 
a

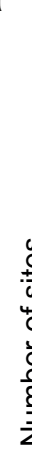

b

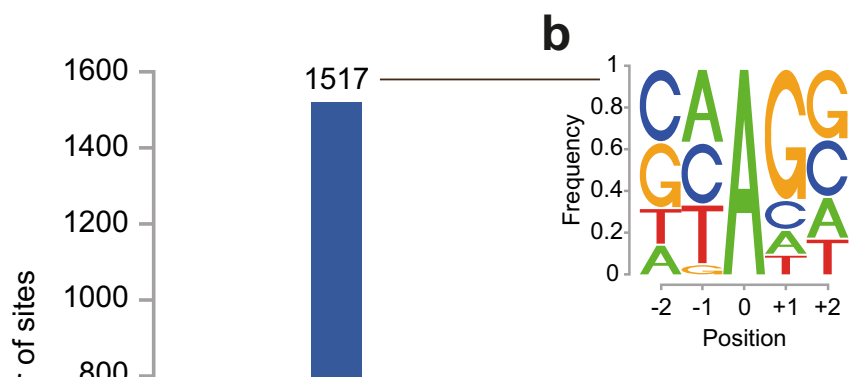

C



25
1006



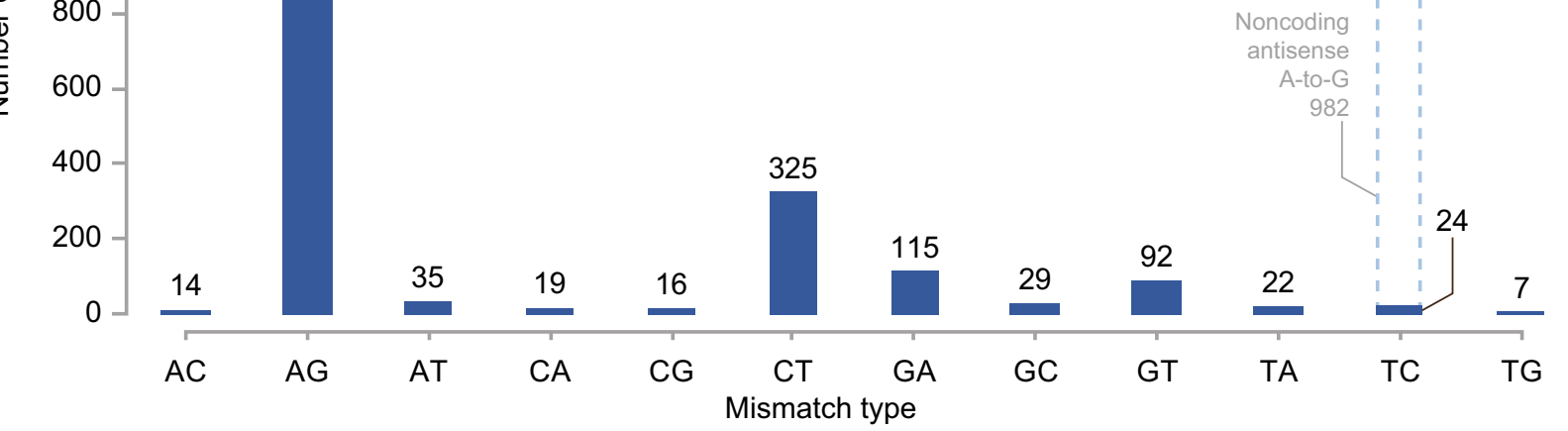

d



e

$\mathbf{f}$
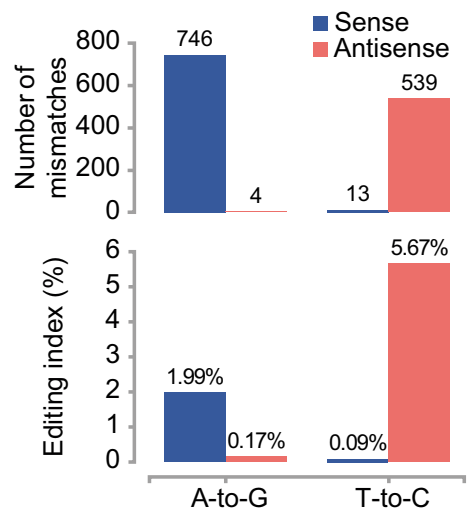

Mismatch type relative to RefSeq CDS annotation

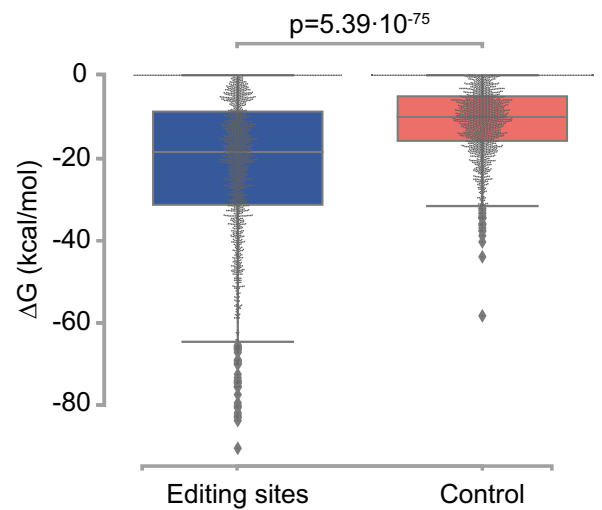

g

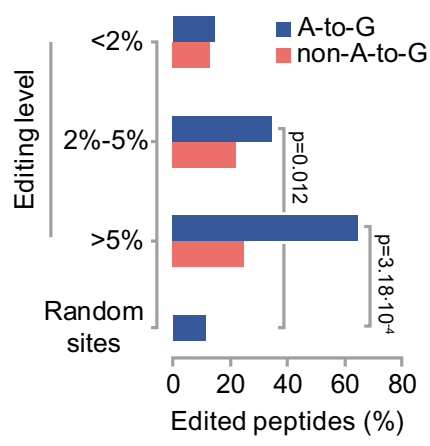

Supplementary Data 8). Of the sites whose editing is conserved in mouse, we found 11 sites in which editing disappears upon ADAR2 knockout (KO), compared to only 3 sites in ADAR1 KO. In $45 / 58$ sites (78\%), editing is suppressed more strongly upon ADAR2 KO (Fig. 3f and Supplementary Data 9$)^{49}$.
Assuming editing in coding sequences is functionally important, we expect the editing levels to be regulated ${ }^{50}$. Looking at the variability of editing levels across individuals (controlling for the sampling noise; "Methods"), we find that weakly edited sites are generally noisy, with standard deviation as high as the mean. 
Fig. 2 The set of 1517 detected sites exhibits ADAR-dependent RNA editing features. a Distribution of the 12 possible substitution types. The ADARderived A-to-G substitution constitutes $\sim 68 \%$ of the total mismatches detected within the coding sequence. We estimate that $\sim 982$ of the $1006 \mathrm{~T}$-to- $\mathrm{C}$ sites ( $98 \%$; dashed light-blue line) are due to non-CDS A-to-I editing events on the non-coding strand (see panel E below). b The local DNA sequence preference surrounding the A-to-G sites is consistent with the previously-reported ADAR preference. $\mathbf{c}$ The T-to- $\mathrm{C}$ sites show the same local sequence preference, reverse-complemented, suggesting that they result from ADAR-mediated editing of RNA expressed from the antisense strand. $\mathbf{d}$ An antisense RNA (red) expressed from a locus that overlaps a protein-coding exon (blue). A-to-I editing produces inosines in the antisense transcripts, manifested as $\mathrm{Gs}$ in RNA-seq reads. Mapping these strand-insensitive reads to the reference genome, antisense editing events appear as T-to- $C$ mismatches with respect to the protein-coding strand. e Top: Analysis of strand-specific RNA-seq samples reveals that 539 out of the 552 T-to-C sites covered by the strandspecific RNA-seq dataset ( $98 \%$ ) were actually A-to-G substitutions on the antisense strand. In comparison, $99.5 \%$ of sites annotated as A-to-G originated from the coding strand, as expected. Bottom: The strand-specific RNA editing index ("Methods") was calculated separately for A-to-G and T-to$\mathrm{C}$ mismatches, showing negligible, indistinguishable from zero, editing level for T-to- $\mathrm{C}$ sites on the coding strand. $\mathbf{f} \mathrm{A}$ swarm plot showing the distribution of free energies for in-silico RNA secondary structures surrounding the 1517 A-to-G sites and the same number of random adenosines as controls. The Ato-G editing sites form significantly more stable structures. P-value by Mann-Whitney test. Box-and-whisker plots show the medians (horizontal lines), upper and lower quartiles (box edges), and $1.5 \times$ the interquartile range (whiskers). g Proteomic mass spectrometry data ("Methods") reveals peptides supporting editing for $65 \%(11 / 17)$ of the sites edited to $>5 \%$ and covered by peptides, compared to $11 \%(18 / 158)$ for random control sites $(p=0.00032$; two-tailed Fisher's exact test). In comparison, peptides supporting editing were found for only $15 \%(13 / 89)$ of the weakly-edited $(<2 \%)$ sites $(p=0.56$, compared to random control sites).

However, the strong sites mostly show a well-regulated behavior (Fig. 3g and Supplementary Data 10).

Clustering of CDS editing sites. It is well known that editing sites in non-coding regions of the genome are clustered ${ }^{51-55}$. Interestingly, we observe here clustering of many sites in the coding region. About half of the sites belong to dozens of clusters, some of which include over 10 sites each (Fig. 4a). Moreover, for many editing sites, we find a putative editing complementary sequence (ECS) that is located up to $15 \mathrm{~kb}$ downstream to the canonical transcript. These structures are mostly overlapping with a paralog gene expressed from the other strand and account for many of the larger clusters (Fig. $4 \mathrm{~b}$ ). We believe these events are due to an extended UTR, not included in the canonical RefSeq transcript, which are known to be expressed in brain tissues ${ }^{56}$. For example, an editing cluster including 19 sites is located within the coding sequence of $c l d n 9$ (Claudin 9). A long ECS is found $1741 \mathrm{bp}$ downstream, overlapping the coding sequence of a paralog gene, cldn6 (Claudin 6). One does observe a cluster of Tto-C sites within this putative ECS, attesting for expression and editing of this region in the CLDN9 coding strand (opposing CLDN6 coding sequence). Consistently, T-to-C mismatches are over-represented in the brain, especially in cerebellum (Supplementary Fig. 5), where the long UTRs are expressed. This suggests a new model for editing regulation, where editing depends on the expression of the longer UTR isoform, which may be tissuedependent (Fig. 4c). The HSPA1L (Heat shock $70 \mathrm{kDa}$ protein 1like) transcript harbors two of the largest clusters found (87 sites). Since this gene is located in vicinity of the highly polymorphic region of the HLA genes in chromosome 6, we have further validated editing in this gene by sequencing matched DNA and RNA blood samples from three individual persons (Fig. 4d; "Methods").

Differential editing of specific recoding sites was reported in multiple diseases ${ }^{57,58}$. We have re-analyzed ${ }^{59-61}$ matched normal and cancer samples of the TCGA dataset ${ }^{62}$ and found eight sites from our set which show a significant and appreciable $(>10 \%)$ change of their editing level in at least one of the nine cancer types studied (Supplementary Data 11). In light of the strong recoding activity in arteries, we have also looked for differential editing in the arteries using GTEx data. Interestingly, of the 27 different diseases and conditions annotated we have found significant and appreciable ( $>10 \%)$ differential editing only in one disease, pneumonia, for which higher editing is observed at five sites (Supplementary Data 12).
Conservation of recoding sites, and signals of adaptation. To gain insight into the evolution of recoding in the mammalian lineage, we have analyzed 5673 RNA-seq samples originating from 21 non-human mammals ${ }^{63-93}$ (Fig. 5a; Supplementary Data 13 and "Methods") and quantified the editing level at the one-to-one orthologous locations for each of the 1517 humandetected sites (when available). Contrary to previous estimates, we find that a sizable fraction of human sites are conserved across species. As many as 835 editing sites ( $55 \%$ of the set) are conserved in at least one of the groups (Fig. 5a). The fraction of conserved sites increases with the (human) editing level (Fig. 5b). For example, $46 \%$ of sites edited to $>10 \%$ and $75 \%$ of sites edited to $>30 \%$ are conserved out of the primates. On the other hand, only 17 sites can be identified as (probably) human-specific ("Methods" and Supplementary Data 14).

It was previously pointed out that the reported editing sites in human coding sequences are depleted in nonsynonymous sites (ratio of nonsynonymous to synonymous editing incidence rates $\left.f_{N} / f_{S}=0.6\right)$ and that editing levels of nonsynonymous sites are generally lower, suggesting an overall deleterious effect ${ }^{94}$. Revisiting this findings with our set of coding-sequences editing sites, we observe neither depletion of nonsynonymous sites nor a lower editing level (abundance $f_{N} / f_{S}=0.93$; not significantly different from unity, $p$-value $=0.20$, proportion test; editing level $p=0.42$, Mann-Whitney test) (Fig. 5c, d). Moreover, taking into account that neighboring synonymous and non-synonymous sites are not mutually independent, and a strong adaptive nonsynonymous site may be accompanied by synonymous (and nonsynonymous) weak "satellite" sites, we looked again at the abundances of sites, focusing on strong $(\geq 10 \%)$ sites and including only the strongest site in each gene. Here, one observes an enrichment and an increased editing level of nonsynonymous editing $\left(f_{N} / f_{S}=1.5 ; p\right.$ value $=0.0028$, proportion test; editing level $p=0.0014$, Mann-Whitney test) (Fig. $5 \mathrm{c}, \mathrm{d}$ ), indicating positive selection. Following Jiang and Zhang ${ }^{95}$, one may distinguish between restorative and diversifying recoding sites. In the former, recoding introduces an amino acid that was encoded in the genome of an ancestral primate species ("Methods"), whereas diversifying editing introduces a novel protein isoform within the considered phylogenetic tree. Comparing the incidence rates and the editing levels of synonymous, restorative and diversifying editing sites, one finds that although diversifying sites are slightly depleted compared to synonymous editing (incidence rate ratio 0.78 , $p=0.016)$, their mean editing level is 1.5 -fold higher $(p=0.019)$ (Fig. 5e, f). Taken together, these results suggest that the 260 strongly-edited $(\geq 10 \%)$ human editing sites are overall adaptive. 
a

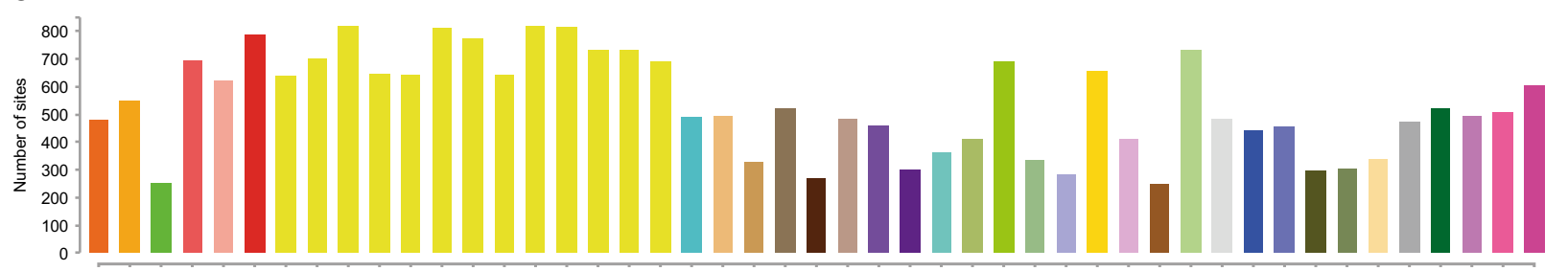

b
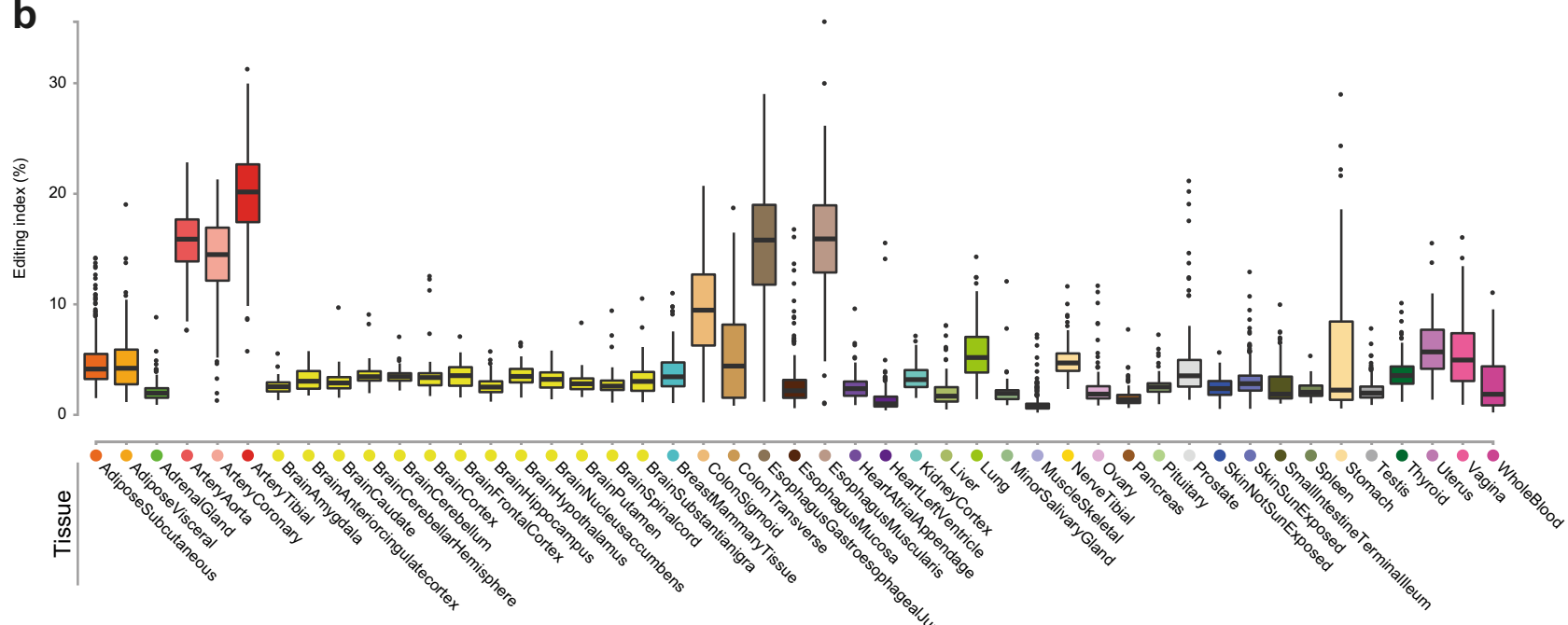

C



f



d

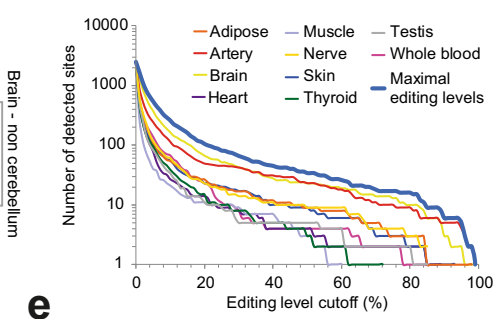

e

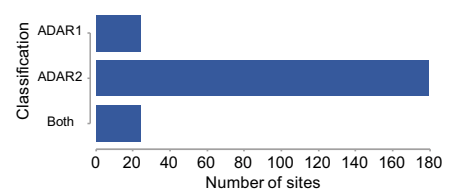

g

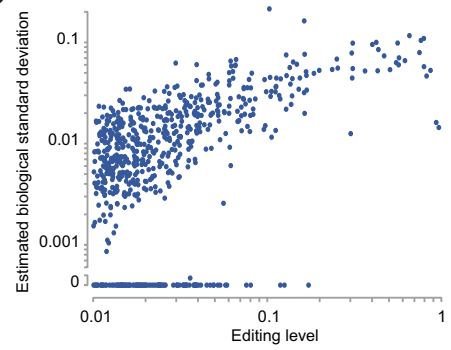

Evolution of editing sites. The detailed comparison of human editing sites to other mammals enables us to examine possible evolutionary routes through which novel editing sites may emerge. Interestingly, even a single mutation may be responsible for the introduction of editing sites. For example, an exon of the ASNS gene pairs with an intronic sequence to form a perfectlypaired $50 \mathrm{bp}$-long dsRNA. This structure is conserved in most primates, but we find no editing (or merely the editing of a single position) out of the Homininae. A single G-to-A mutation in the intron resulted in an $\mathrm{A}: \mathrm{C}$ mismatch specific to the Homininae.
Such a mismatch flanked by long dsRNAs is known to be a preferred ADAR target ${ }^{52}$. Consistently, the mutated intronic $\mathrm{A}$ is strongly edited, and four addition editing sites appear in the vicinity of the exonic C (Fig. 6a). On the other hand, a few mutations can abolish strong editing sites, as is demonstrated by the NEIL1 example. Two adenosines are strongly edited (79 and $94 \%$ ) in NEIL1 coding sequence in human, but not in rodents (no editing in rat; 9 and $0.5 \%$ editing in mouse). It was previously suggested that the strong editing is primate-specific and depends on a neighboring Alu sequence ${ }^{96}$. However, these two sites are 
Fig. 3 Tissue-dependence and ADAR-specificity of CDS editing sites. a Number of sites detected and edited to $>1 \%$ per-tissue (out of the total 1517). The highest numbers of sites are observed in the nervous system, arteries, and lung. b Box-and-whisker plots, depicting the distribution of per-tissue CDS editing index values reveal that although the number of edited sites in the brain is large, CDS editing activity (number of deamination events) is comparable to most other tissues and is much lower than in arteries, colon or esophagus. Box-and-whisker plots show the medians (horizontal lines), upper and lower quartiles (box edges), and $1.5 \times$ the interquartile range (whiskers). See Supplementary Data 1 for the number of biologically independent samples per tissue. c t-SNE 118 dimensionality-reduction analysis ("Methods") reveals highly distinctive CDS editing pattern in cerebellum, arteries, testis, and noncerebral brain regions. d The number of sites detected depends strongly on the editing level cutoff. For all tissues, about half of the detected sites are weakly edited $(<1 \%)$. e Analyzing previously published RNA-seq data from ADAR1- and ADAR2-overexpressing human cell lines, we classify 227 of the sites (well covered and sufficiently edited in cell lines data) based on the editing enzyme. The vast majority of these are targeted by ADAR2 (see Supplementary Data 8). (f) Analyzing previously published RNA-seq data from ADAR1 and ADAR2 knockout mice ${ }^{14,49}$, we calculate the ADAR Influence value (see Methods) for 58 sites conserved in mouse (all sites with $>5 \%$ in wild type mouse and significant, Fisher $p<0.05$, difference between wild type and the double-knockout mouse, excluding the GRIA2 site that was genomically edited to G in the double-knockout) (See Supplementary Data 9). The heatmaps depict the editing levels and the ADAR Influence value (darker: ADAR1, lighter: ADAR2). $\mathbf{g}$ The biological variability ("Methods") in editing levels across individual donors. Many of the strongly edited sites exhibit standard deviations much smaller than the mean, implying regulation on the RNA editing activity. Source data are provided as a Source Data file.

extensively edited in the Alu-lacking sheep (91 and 94\%). The secondary structure is similar in all mammals, but a few mutations result in the appearance of two asymmetric internal loops in rat (compared with one in human, and a larger symmetric loop in sheep), which may be responsible for the dramatic suppression of editing (Fig. 6b). Finally, a human specific editing site appears following a G-to-A mutation within an exon of the sema5b (Semaphorin 5B) gene (Fig. 6c). The mutation is located within a region that forms a dsRNA structure, and changes a $\mathrm{G}: \mathrm{C}$ pair into an A:C mismatch, which is favorably edited.

\section{Discussion}

Recoding by A-to-I editing provides an additional layer of the complex regulation of the proteome. Mapping the landscape of recoding sites enables future studies to reveal the full functional impact of recoding in mammals, across previously unstudied tissues, pathways, and pathological conditions. It also provides a better understanding of the structural and sequence determinant underlying recoding efficiency and regulation which may be then utilized for an improved design of ADAR-based RNA engineering strategies.

Most sites in our list are weakly edited. Furthermore, the number of detected sites depends strongly on the editing level cutoff, and there are thousands of additional sites edited to $<1 \%$ in all tissues examined. These are less likely to be functionally important. However, it is possible that sites appearing to be weakly edited when averaged over a tissue under normal conditions, exhibit much higher editing levels under specific conditions, in specific subpopulations of cells ${ }^{46}$, or even at a single-cell level ${ }^{97,98}$. Combining large-scale single-cell sequencing data with the editing detection method presented here could reveal the full scope of recoding and its functional impact.

\section{Methods \\ Human RNA-sequencing data. RNA-seq samples from the Genotype-Tissue Expression (GTEx) project $^{34}$ were used as the core input for our de-novo RNA editing detection pipeline. Raw data (76bp-long, paired-end reads) were accessed via the database of Genotypes and Phenotypes (dbGaP; Study Accession: phs000424.v8.p2) and FASTQ files were downloaded via Sequence Read Archive (SRA) using SRA Toolkit (v2.8.0). We used the raw reads and not the alignment files available from GTEx, as they include multi-loci mapped reads, which hinder de-novo discovery of editing sites. A total of 9125 RNA-seq samples from 548 donors and 47 tissues were analyzed (Supplementary Data 1).}

Nonhuman RNA-sequencing data. Nonhuman mammalian RNA-seq datasets were collected from varied sources ${ }^{63-93}$ to test the conservation of the de-novo detected human editing sites across the mammalian lineage. Altogether, 5673 RNA-seq samples (Supplementary Data 13) from 21 different nonhuman species (Supplementary Data 15) were analyzed.
Reads alignment and BAM processing. Raw reads were mapped to the reference human genome using STAR RNA-seq aligner (vSTAR_2.5.2b) ${ }^{99}$ as follows: The reference genome (hg38; analysis set version) was downloaded from the UCSC Genome Browser ${ }^{100}$ (http://hgdownload.soe.ucsc.edu/goldenPath/hg38/bigZips/ analysisSet/), and indexed using default parameters and no annotation files. The reads were then aligned in a per-sample basic two-pass mode (-twopassMode Basic), keeping only uniquely mapped reads (-outFilterMultimapNmax 1). The "-outSAMattributes All" option was used in order to output the MD tag which is required for the detection of mismatches in later steps.

Duplicated reads were marked using Picard Tools MarkDuplicates program (v2.6.0-SNAPSHOT; http://broadinstitute.github.io/picard/), later to be ignored by our pipeline. Next, BamUtil clipOverlap (v1.0.13; https:/github.com/statgen/ bamUtil) ${ }^{101}$ was applied to clip one of the reads in pairs where the two pair-mates overlap. Different RNA-seq samples originated from the same donor and tissue (technical duplicates) were merged into a single BAM file using SAMtools marge (v0.1.18; http://www.htslib.org/) ${ }^{101}$.

Nonhuman samples were processed as described for human and were mapped to the respective reference genomes (Supplementary Data 15).

Read-level detection of mismatches. BAM files were converted into SAM format using SAMtools view (v0.1.18), and were analyzed to detect all mismatches between the reads and the reference genome. We excluded mismatches that were located at the ends of reads or alignments ( $5 \mathrm{bp}$ form each end) or near splice-sites ( $4 \mathrm{bp}$ ), adjacent to a homopolymeric sequence in a length of $5 \mathrm{bp}$ or more (upstream or downstream), or in positions with low base quality score $(q<30)$. In addition, we excluded reads of low quality ( $25 \%$ of the read with $q<20)$, and those which failed the platform/vendor quality checks, were not mapped as a proper pair, were PCR or optical duplicates, or included three or more mismatches of more than two distinct types. Eventually, a list of mismatches of all twelve possible substitution types for all donors in every available tissue was obtained. For each mismatch the number of reads supporting it and the total number of covering reads (i.e., total depth) were reported. In addition, the coverage by reads mapped to the positive strand of the reference genome and the number of those reads supporting the mismatch were recorded.

\section{Annotation-based filtering of mismatches. Our analysis focused solely on} protein-coding regions within curated transcripts, as annotated by RefSeq (records with an NM_ prefix; see below). Regions, where both strands are annotated as RefSeq coding exons (total length: 5639 bp), were discarded. As GTEx RNA-seq data is not strand-specific, we assumed the expressed strand to be the annotated coding strand. To minimize the extent of erroneously-called substitutions we excluded from the analysis the following genomic regions or locations that are suspected to introduce false-positive detections of RNA editing sites: (i) Common genomic single-nucleotide variations in dbSNP150 or dbSNP147, (ii) 50 bp regions around common insertions and deletions (indels) in dbSNP150 or dbSNP147. (iii) Rare SNPs (found in $<1 \%$ of the population) where the reference genome includes the rare allele according to the alleles frequencies reported in either the 1000 genome project ${ }^{102}$ or the Trans-Omics for Precision Medicine (TOPMed; https:// www.nhlbiwgs.org/) project using dbSNP150 and dbSNP146. In addition, "known issues" reported directly by dbSNP (https://ftp.ncbi.nih.gov/snp/organisms/ human_9606_b150_GRCh38p7/known_issues/) were excluded, as well as $50 \mathrm{bp}$ regions flanking indels in this list. In all the three lists above (i-iii) we used the union of the newer dbSNP150 and an older version (147 or 146), as we found some false-positive detections to be missing from the newer dbSNP version. (iv) repeatMakser-annotated rDNA repeats were excluded, as there are multiple nearlyidentical such genomic regions. Therefore, reads aligned to these regions are prone to misalignments. In addition, repeatMakser-annotated $A l u$ regions were excluded. In contrary to the aforementioned regions that may be prone to false-positive 
a

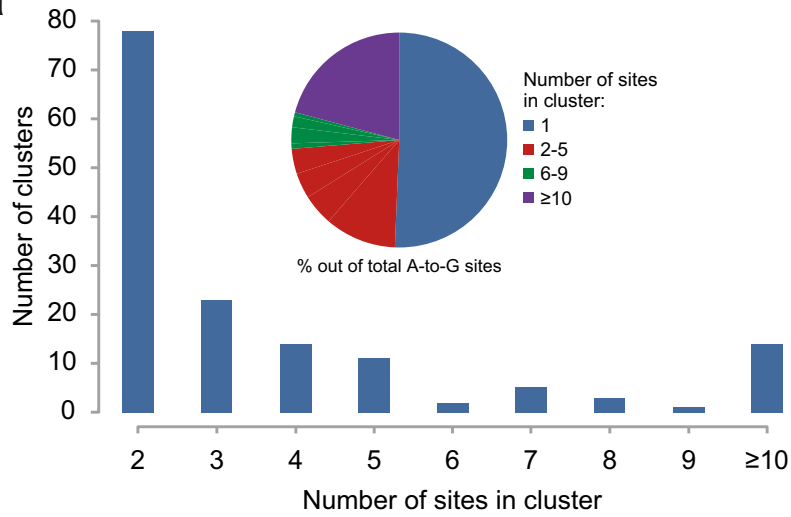

b

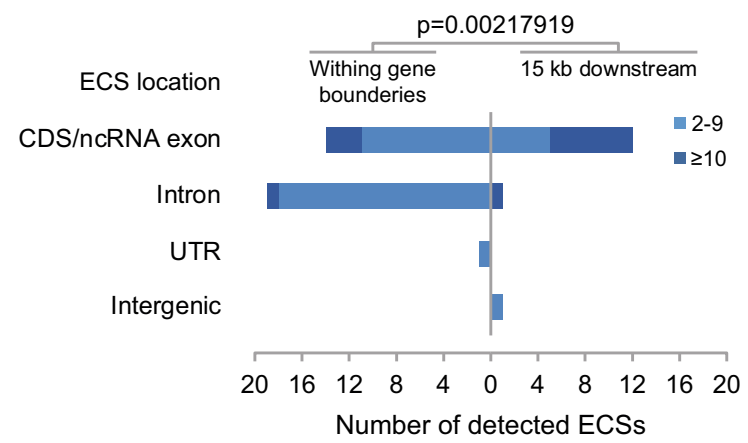

C
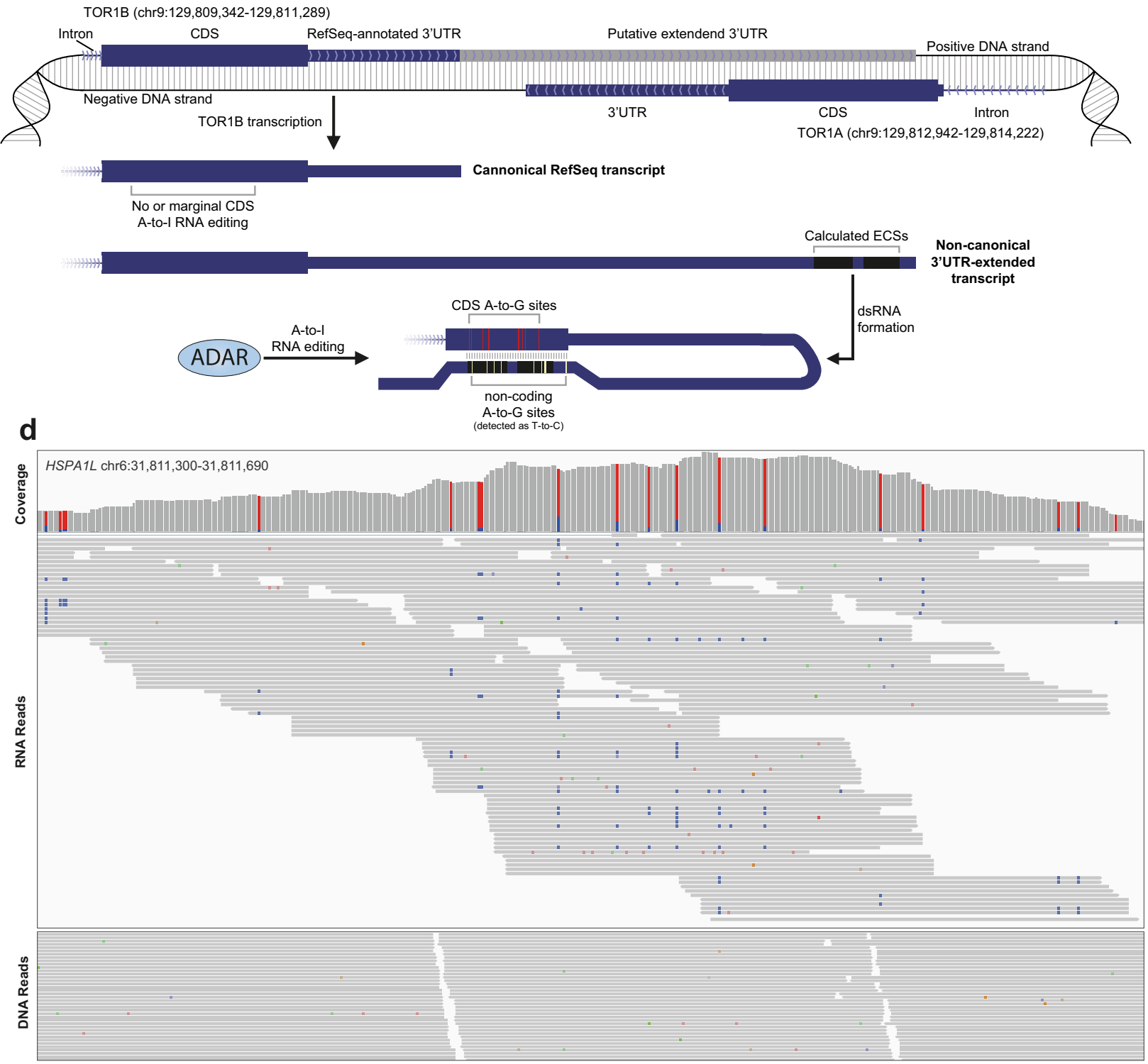

detections, Alu elements are actually known to be heavily edited ${ }^{44,103}$, and were excluded to prevent a bias in the accuracy calibration of the pipeline due to the numerous editing events in these regions.

Original dbSNP tables, RefSeq and repeatMakser annotations were downloaded from the UCSC genome browser website ${ }^{100}$ (http://hgdownload.soe.ucsc.edu/ goldenPath/hg38/database/). The RefSeq and the repeatMasker tables are updated as of August 8, 2017 and January 1, 2018, respectively. These filtered regions sum up to $2.1 \%$ out of the RefSeq annotation that was used.
BLAT-based mismatches filtration. Many mismatches result from erroneous mapping of reads to a highly similar locus in the reference genome. To clean these, we used a sliding window approach, looking each time at a $76 \mathrm{bp}$-long subsequence (to match reads' length) of all RefSeq coding sequences with a step of $19 \mathrm{bp}$ ( $25 \%$ of the read length). These $76 \mathrm{bp}$-long subsequences were aligned to the reference genome using BLAT (BLAST-like Alignment Tool) ${ }^{104}$, in the search of highlysimilar genomic regions (alignment length $\geq 61 \mathrm{bp}, \geq 94 \%$ identity, no indels in query), mainly due to duplicated genomic regions and processed pseudogenes. 
Fig. 4 Many of the CDS editing sites are clustered. a Bar plot: Distribution of editing clusters by size. Pie chart: Relative abundance of editing sites by cluster size. b Editing requires a dsRNA secondary structure, often depending on a distant editing complementary sequence (ECS). Distribution of putative ECSs location ("Methods") shows that smaller clusters tend to depend on an intronic ECS, while the ECS for large clusters ( $\geq 10)$ is often found out of the annotated gene boundaries, overlapping an exon of a neighboring downstream gene. c Editing due to a downstream reversely-oriented paralog gene, overlapping an extended 3'UTR. The TOR1B gene contains a 10-site RNA editing cluster in its coding sequence. The corresponding ECS is a closely-related sequence that overlaps a coding exon within the neighboring paralog TOR1A gene (genomic coordinates of the last exon of both genes are shown). Presumably, the 3'UTR of edited TOR1B transcripts is longer than that of the Refseq canonical transcript, extending to overlap the coding sequence of Tor1a (on the opposite strand). These extended-3'UTR variants of the Torlb transcripts contain two highly-similar reversely-oriented sequences, and can form a long and stable dsRNA structure, resulting in extensive editing of both the CDS (red lines) and the TOR1A-overlapping 3'UTR (yellow lines). A 14-site cluster of T-to-C sites is detected in the coding sequence of TOR1A, a hallmark of A-to-I editing in antisense transcripts (see Fig. 2d). See Supplementary Data 3 for further evidence for expression and editing of the extended UTR of TOR1B. d Editing at one of the large clusters within the HSPA1L transcript. Up: pile-up of the RNA reads' coverage, 19 different editing sites are observed in this 391 bp-long segment of the editing cluster (red and blue bars stand for A and $G$ fractions, respectively). Bottom: Matched RNA and DNA sequences (presented is the pooled data for the three individuals, for simplicity). Editing events (A-to-G mismatches) are shown in blue (different colors represent other type of mismatches). The absence of A-to-G mismatches in the matched DNA samples and lack of linkage between neighboring sites both support the sites being RNA-edited.

a

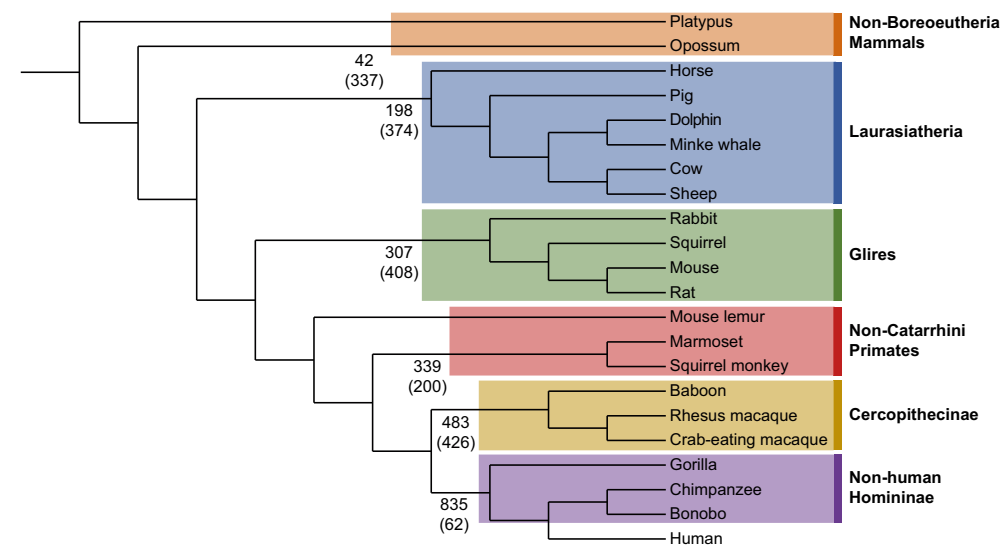

b



C

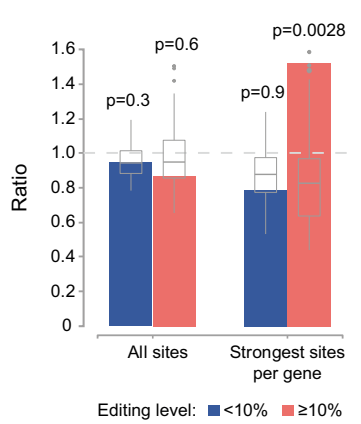

d

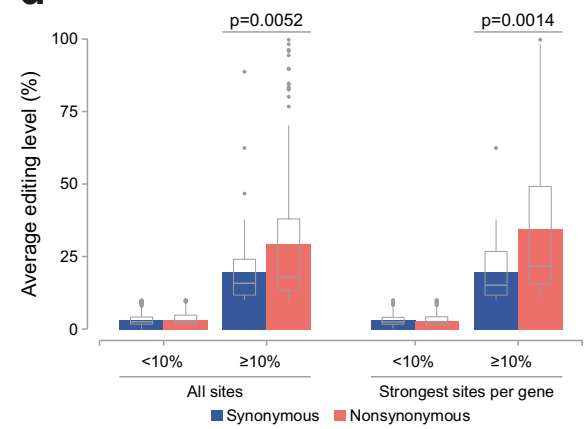

e

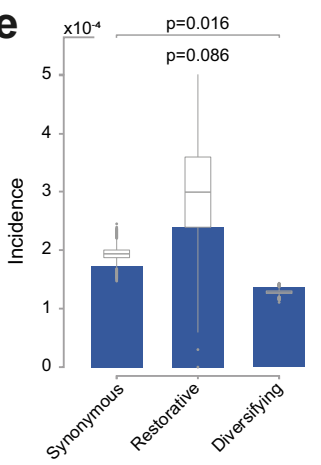

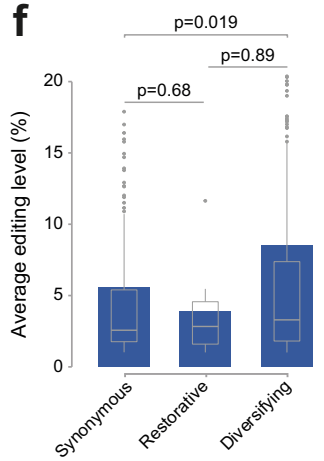

Fig. 5 Evolution, conservation, and signs for adaptation in CDS-editing sites. a Phylogenetic tree of the mammalian species analyzed (branch lengths not to scale). For each of the six evolutionary groups, numbers on left present the number of sites conserved between the human and the last common ancestor (LCA) of the group, and, in parentheses, number of human sites which are determined as not edited in the group ("Methods"). b Fraction of evolutionary-conserved sites whose (human) editing level is above a given cutoff, as a function of this cutoff. c Ratio of incidence rates for synonymous and non-synonymous sites is approximately unity (dashed gray line), for weak $(<10 \%)$ and strong $(\geq 10 \%)$ sites alike. For the strongest site in the gene, weak nonsynonymous editing sites are slightly depleted while strong nonsynonymous sites are enriched, suggesting positive selection and adaptivity. Box-andwhisker plots represent the distribution of ratios over $10^{6}$ random As, controlling for the \pm 1 bp nucleotide context. $\mathbf{d}$ Mean (colored bars) and distribution (box-and whisker) of editing levels. Number of sites: 879 nonsynonymous and 378 synonymous weak sites; 177 and 83 strong sites. Considering the strongest site per gene only: 408 nonsynonymous and 211 synonymous weak sites; 105 and 28 strong sites. e Sites were classified into three categories based on the reconstructed amino acid encoded by ancestral primates ("Methods"): synonymous, restorative (i.e., recoding results in an amino acid encoded by ancestral primate), or diversifying sites (otherwise). The incidence rate ("Methods") is higher for restorative editing (not significant) and slightly lower for diversifying sites, relative to synonymous ones. Box-and-whisker plots present distribution of ratios over $10^{6}$ random As, controlling for the \pm 1 bp nucleotide context in each of the three categories. $\mathbf{f}$ Mean (colored bars) and distribution (box-and whisker) of editing levels for 581 diversifying, 8 restorative, and 239 synonymous sites. Box-and-whisker plots show the medians (horizontal lines), upper and lower quartiles (box edges), and $1.5 \times$ the interquartile range (whiskers). P-values by randomization test $(\mathbf{c}, \mathbf{e})$ or two-sided Mann-Whitney test (d, $\mathbf{f})$. Source data are provided as a Source Data file. 
a

ASNS (chr7:97,869,000-97,870,299)

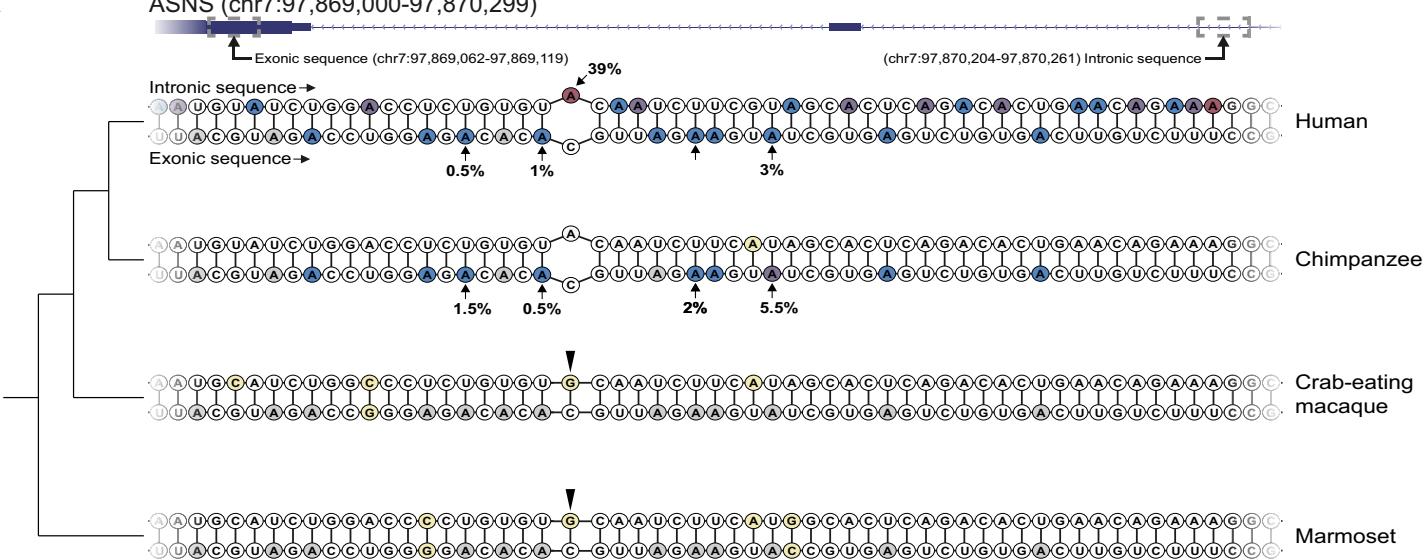

b

NEIL1 (chr15:75,353,653-75,353,822)



C

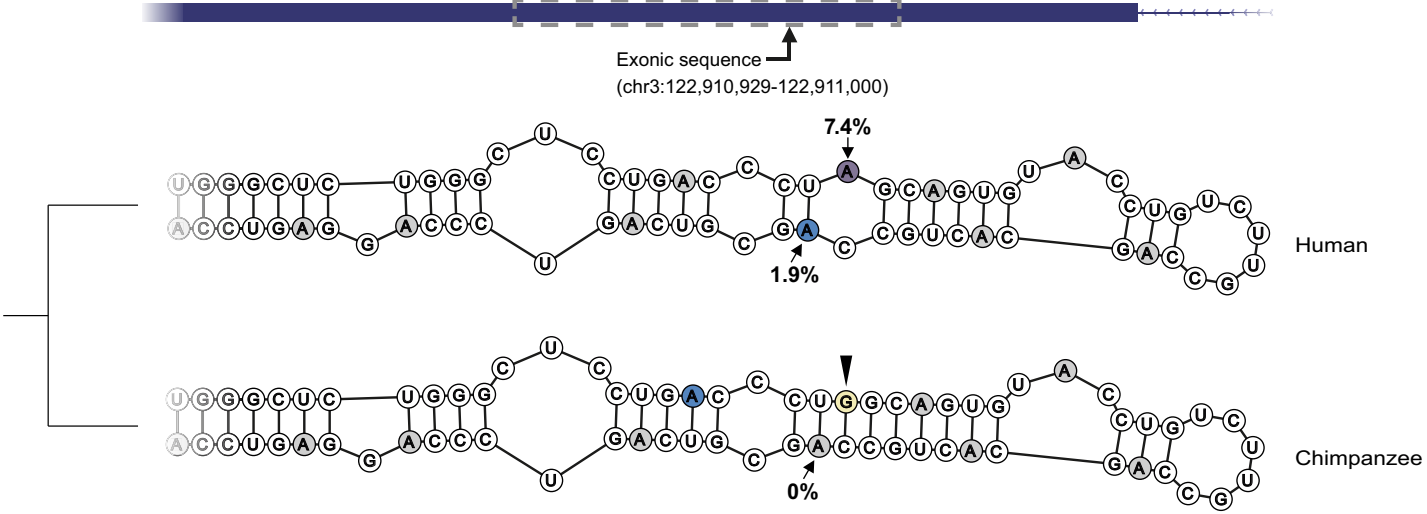
- Altered dsRNA structure
Editing levels (\%):
(A) $[5,20)$
$[80,100]$
(N) Genomic variant
(A) $[0,0.1)$
(A) $[20,60)$
(A) Not available
- Indel
(A) $[0.1,5)$
(A) $[60,80)$

Mismatches found by these BLAT alignments, are likely to appear as mismatches between RNA-seq data and the reference genome, due to misalignments of sequencing reads originating from one genomic locus to another, highly-similar, locus. Thus, these mismatches were discarded from the above list of potential editing sites. A-to- $\mathrm{G}$ mismatches were detected in $\sim 0.5 \%$ of all adenosines in the RefSeq annotation.
Statistical model and score calculation. To decide whether a specific genomic position is an editing site, we employed a statistical model that integrates the data from all donors for each tissue, using a maximum likelihood approach. For each donor, three binomial tests and three $p$-values are evaluated per site, to assess the likelihood of the observed total coverage $C$ and number of reads supporting a mismatch $E$, assuming editing does not occur at this site: 
Fig. 6 Species-specific dsRNA structures contribute to the formation of novel RNA editing sites. a Several hominid-specific exonic RNA editing sites were introduced to the Asns gene following a single G-to-A genomic substitution modifying the RNA secondary structure. Two uppermost sequences show a few CDS adenosines being edited in human and chimpanzee. These editing events are not conserved in more distant primates. In non-hominid primates (two lowermost structures), the RNA structure is very similar, except for a single hominid-specific mismatch (internal loop) feature, resulting from a genomic G-to-A substitution in the intronic ECS of hominids. b The extensive editing in NEIL1 CDS seen in primates (top) is conserved in some nonprimates as well (bottom). However, editing is much weaker in rodents (middle). Mutations in the rat sequence alter the secondary structure, while the sheep structure is more similar to the human one. c The emergence of human-specific RNA-editing site. A human-specific G-to-A substitution results in an $A: C$ mismatch within a dsRNA structure already present in the ancestral hominid. This newly introduced adenosine is efficiently deaminated by the ADAR enzymes, and an additional weaker site appears in the complementary sequence. In each of the three panels, the top track shows the locus of the edited CDS region and the ECS (gray dashed-line boxes) within the corresponding gene (blue boxes - exons; lines with arrowheads - introns); the bottom track shows the corresponding calculated secondary structures. The topology of the phylogenetic tree for the species presented is shown on the left (branch lengths not to scale). Species names are denoted on the right. Major structure alternations along with indels and single nucleotide substitutions (relative to human) are indicated. Adenosines in all structures are annotated in different colors based on their calculated RNA editing levels (pooling all brain samples available, per species; Gray - no detectable editing, blue - low editing levels; red - high editing levels). Editing levels were not assessed for sites covered by $<50$ reads.

1. The probability that the retrieved $C$ and $E$ are merely due to sequencing errors, given a maximal probability of base calling sequencing error of $E_{\text {error }}=10^{-3}$ (as set by the minimal Phred quality score to call substitution of $q=30$ ):

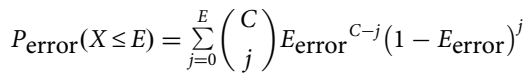

2. The probability that the retrieved $C$ and $E$ represent a rare heterozygous SNP, given an expected frequency of the alternative allele $E_{\text {hetero }}=0.5$ and an a-priori probability of such a genomic event of $p_{\text {hetero }}=10^{-4}$ :

$$
P_{\text {hetero }}(X \leq E)=\left(\sum_{j=0}^{E}\left(\begin{array}{l}
C \\
j
\end{array}\right) E_{\text {hetero }}{ }^{j}\left(1-E_{\text {hetero }}\right)^{C-j}\right) \cdot p_{\text {hetero }}
$$

phetero was estimated based on ${ }^{102}$. The typical number of SNPs in the coding sequence ranges between 21.4 and $26 \mathrm{k}$ for five distinct human populations. Of these, $1-4 \%$ are rare SNPs (prevalence $<0.5 \%$ ). As the total length of RefSeq CDS is $34 \mathrm{Mbp}$, the probability to have a SNP at a given position can be as high as $\left(2.6 \times 10^{4} / 3.4 \times 10^{7}\right) \times 0.04=3.06 \times 10^{-5}$. As we defined rare SNPs as those with prevalence $<1 \%$, the probability could still be higher. Adopting a conservative approach, we have therefore set $p_{\text {hetero }}=10^{-4}$.

3. The probability that the retrieved $C$ and $E$ represent a rare homozygous SNP, Given an expected frequency of the alternative allele $E_{\text {homo }}=1$ and an estimatedprobability of such genomic event of $p_{\text {homo }}=\left(p_{\text {hetero }}\right)^{2}=10^{-8}$ :

$$
P_{\text {homo }}(X \leq E)=\left(\sum_{j=0}^{E}\left(\begin{array}{c}
C \\
j
\end{array}\right) E_{\text {homo }}{ }^{j}\left(1-E_{\text {homo }}\right)^{C-j}\right) \cdot p_{\text {homo }}
$$

We choose the most likely of the above three explanations and adopt the lowest of these three $p$-values. Had this been a bona fide $p$-value, one could have then incorporated the multiple $p$-values (one per each donor) into one statistic using Fisher's method, and define a score $S$ per site based on the full data from $N$ donors to be (natural $\log$ ):

$$
S=-2 \sum_{i=1}^{N} \log \left(\min \left[P_{\text {error }_{i}}, P_{\text {herero }_{i}}, P_{\text {homo }_{i}}\right]\right)
$$

This statistic should then have a $\chi^{2}$ distribution with $2 N$ degrees of freedom. However, as we allow for more than one test per site and choose the most likely possibility, the chosen $p$-value per donor is not truly a $p$-value (in the sense that the values are not uniformly distributed along the unity interval given the null hypothesis of no editing). Therefore, $S$ does not follow the $\chi^{2}$ distribution. Yet, our approach overestimates the true $p$-value, and estimating the likelihood of getting values as high as $S$ by the $\chi^{2}$ distribution would provide an underestimate of the statistical significance. Note, however, that the statistical significance obtained is only correct if one assumes the mismatches of different reads to be independent. De facto, we have found that many of the false-positive detections are due to systematic misalignments and other factors not satisfying this independence assumption. To avoid confusion and misinterpretation, we therefore chose not to assign a $p$-value to each site, but use the score only as a filtering step. Mismatch sites with a score $S<2 N$ were discarded ( $2 N$ being the mean of the distribution).

Another $p$-value-based filtration was aimed to eliminate several sorts of errors that are specific to one sequenced-strand. During the sequencing protocol, the original RNA fragments are reverse-transcribed into double-stranded cDNA which is then amplified. The sequencing machines sequence both strands of these cDNA fragments, regardless of the original expressed strand. Therefore, an RNA editing event should be manifested as a mismatch showing up in both strands of the cDNA. In contrast, mismatches due to technical sequencing and amplification errors, are often strand specific ${ }^{22}$. Thus, we have pooled the data from all samples and applied a binomial test to the reads aligned to each of the genomic strands, separately, requesting that both tests result in a significant $p$-value rejecting the possibility of sequencing errors (both $p$-values $<\exp (-N / 4)$ ).

Cluster-based filtering. Clusters of mismatch sites, i.e., multiple mismatch sites of different substitution types (e.g., A-to-G and C-to-A, etc.) at the same locus, might point to problematic read alignments (e.g., mapping of reads due to a polymorphic pseudogene to its original gene) leading to false-positive detections. To filter these, we calculated the positions of all mismatches relative to the mature mRNA sequences using Annovar (v2018Apr16; https://doc-openbio.readthedocs.io/ projects/annovar/en/latest// ${ }^{105}$ with RefSeq-transcripts built database. Using BEDtools cluster (v2.26.0) ${ }^{106}$, we joined pairs of mismatch-positions at a distance $\leq 100$ bp apart into clusters, iteratively. Clusters containing more than one type of mismatch were discarded.

Whole-exome-sequencing-based filtering. To further clean up our list from sites with a genomic origin, we analyzed WES data, deposited as a part of the GTEx dataset. Additionally, this analysis may also filter out candidate sites that resulted from alignment errors. A total of 609 WES samples from 602 distinct donors (mostly from whole blood specimens) were used (Supplementary Data 16). Matching WES data was available for 504 of the 548 RNA-seq donors. The other 98 unmatched WES data were used for the analysis as well. We mapped the WES data using the same pipeline applied to the RNA-seq samples, and calculated the WESmismatch level at each of the putative editing sites. Sites with insufficient $(<100)$ WES reads or a WES-mismatch level exceeding $0.1 \%$ (namely, the maximal expected sequencing error rate) were discarded.

Recalculating editing levels and editing-level-based filtration. To further reduce noise in our set of sites we also excluded sites with low editing level, as sites in which a very small fraction of the transcripts were edited are assumed to have only a limited contribution to functionality. To correctly assess the per-site editing levels in each of the tissues, candidate sites from the 47 tissues were combined to a single list. Next, the total coverage and the number of reads supporting the mismatch (i.e., edited reads) were calculated from the original unfiltered BAM files

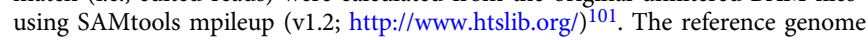
was not supplied and the following options were applied: -B -Q 0 -d $10000000-\mathrm{ff}$ SECONDARY,UNMAP,DUP. The per-site-per-sample (i.e., donor in a certain tissue) coverage data were summed. Sites that did not exhibit an editing level $\geq 1 \%$ in at least one tissue were discarded. The resulting 1517 A-to-G sites, their annotation, and editing level per tissue, appear in Supplementary Data 2.

Maximal editing levels. The maximal editing level was calculated separately for each site. It was defined as the maximum editing level across all tissues with coverage $\geq 100$ reads. Sites' maximal editing level was used in all non-tissue-specific analyses as a representative editing level value unless otherwise stated.

RNA editing index. The editing index is defined as described in ${ }^{44}$ for all GTEx samples. Briefly, for a chosen set of A-to-G editing sites, mpileup data was used to determine the number of observed matched As and the number of mismatching Gs at the sites. The index is the percent of Gs out of the number of As and Gs combined. The editing index values were calculated separately for each tissue type unless stated otherwise. The T-to-C index was assessed in the same manner.

Coverage calculation in nonhuman samples. Genomic coordinates of the sites orthologous to the 1517 human RNA editing sites in the 21 nonhuman reference genomes were found using the liftOver program (http://hgdownload.soe.ucsc.edu/ 
admin/exe/linux.x86_64/liftOver) with default parameters and the appropriate conversion files (*.over.chain.gz files). Then, liftOver was used again to reconvert nonhuman coordinates back to the human reference genome, to verify these are reciprocal best hits, thus a common proxy for real orthologs. For each species, unmapped records, and records for which the orthologous site was not mapped back to the original human coordinates were discarded. Whenever the nucleotide at the orthologous genomic position in a given species was an adenosine, total coverage and a total number of edited reads were calculated for each biological sample, using mpileup as described in the paragraph above.

Identification of conserved RNA editing sites. We used NCBI taxonomy (https:// www.ncbi.nlm.nih.gov/taxonomy) to construct a phylogenetic tree for human and the 21 nonhuman mammals. Inner nodes exhibiting multifurcation were manually bifurcated according to UCSC genome browser 100-way-multiple-sequence-alignment-based calculated phylogenetic tree (http://hgdownload.soe.ucsc.edu/goldenPath/ hg19/multiz100way/hg19.100way.scientificNames.nh). The tree was visualized using iTOL (ver. 5.6.3) online tool ${ }^{107}$

In order to gain sufficient sequencing coverage and minimize multiple-testing (thus increasing statistical power), the 21 individual mammal species were grouped into 6 distinct groups as follows: nonhuman-Homininae, Cercopithecinae, nonCatarrhini primates, Glires, Laurasiatheria, and non-Boreoeutheria mammals (Supplementary Data 15). Due to their different editing pattern, samples in each group were further classified into two categories: brain and non-brain samples (Supplementary Data 13). The per-site values of total coverage and number of supporting reads were summed over all samples in each of the six groups of organisms and each category, resulting in 12 different lists. A binomial test was applied for each site, with the null hypothesis that the site is not edited in a given nonhuman group and category, and possible mismatches can be attributed to random sequencing errors (with a rate of $10^{-3}$, corresponding to $q=30$ ). The resulted $\mathrm{p}$-values were adjusted using Benjamini and Hochberg's False Discovery Rate $(\mathrm{FDR}=0.001)$ correction, for each of the 12 lists, separately. A human RNA editing site was considered conserved in a certain nonhuman group if it was detected in either brain or non-brain tissues. On the other hand, a site that was not found to be conserved and exhibited a non-significant $>0.05$ FDR and a total coverage $\geq 500$ in either brain or non-brain categories was considered nonconserved in this group of species (Supplementary Data 2). To determine the emergence of editing at a given site along mammalian evolution, we look for the last common ancestor (LCA) of human and the most distant mammal exhibiting RNA editing, for which the editing in the most recent outgroup (of the descendants of the LCA) is non-conserved. This LCA is considered to be the one at which the editing of a site has emerged.

To search for human-specific editing sites (Supplementary Data 14), we looked for either (i) edited human adenosines where the reconstructed nucleotide in the ancestral Hominin sequence is not an adenosine (and therefore not edited), or (ii) human sites exhibiting an editing level $>2 \%$ in cerebellum and cortex that were deemed non-conserved (as defined above) in nonhuman-Homininae and in one of Cercopithecinae or non-Catarrhini primates.

Calculating the relative frequency of synonymous and nonsynonymous editing sites. To estimate the extent to which our detected A-to-G sites are positively selected throughout evolution we used an approach that largely follows ${ }^{94}$. First, choosing the longest protein-forming transcript per gene from RefSeq annotation (see above), we counted CDS adenosines whose potential editing into inosines would have resulted in synonymous and nonsynonymous changes $(S=2,512,451$ and $N=6,185,931$, respectively). Then, we calculated the ratios of the number of actual editing sites in our list to the number of potential sites, resulting in $f_{\mathrm{S}}=1.83 \times 10^{-4}$ and $f_{\mathrm{N}}=1.71 \times 10^{-4}$ for synonymous and nonsynonymous editing, respectively. As weak editing sites are expected to have a lesser functional impact, we have repeated the calculation for weak $(<10 \%)$ and strong $(\geq 10 \%)$ sites separately. In addition, for each of the four sets of weak and strong, synonymous and non-synonymous, sites, we constructed $10^{6}$ sets of 1517 randomly chosen CDS adenosines, with the same distribution over chromosomes and sequence contexts ( 1 bp downstream and upstream), and calculated the mean and SD over the $10^{6}$ random replications. $P$-values were estimated by a randomization test, checking what proportion of the $10^{6}$ random sets produce a value equal or higher than the one measured in the actual data. Finally, we repeated the analysis, taking into account only the most strongly edited site per gene.

\footnotetext{
Ancestral state reconstruction for RNA editing sites and classification to restorative and diversifying. Ancestral state reconstruction was performed similarly to ${ }^{95}$ using sequences of the following organisms: Homo sapiens, Pan troglodytes, Pan paniscus, Gorilla gorilla, Macaca mulatta, Macaca fascicularis, Papio anubis, Callithrix jacchus, Saimiri boliviensis, Mus musculus and Bos taurus while the latter two served as outgroups (Supplementary Fig. 6). Human RefSeq accession numbers of each gene that harbors one or more editing sites were converted to Ensembl IDs via Ensembl API (http://www.ensembl.org/info/docs/ index.html), retaining only genes having one-to-one orthologous genes in all the indicated species. Next, mRNA and protein sequences of the Ensembl canonical transcript were retrieved for each ortholog. Clustal Omega (Ver. 1.2.1) ${ }^{108}$ with
}

default parameters was then used to generate a per-gene multiple sequence alignment of the orthologous protein sequences. Next, the protein sequence alignments were converted to the corresponding codon alignments using PAL2NAL ${ }^{109}$, and these were then used to reconstruct the ancestral transcripts sequences in a maximum-likelihood-based analysis utilizing the codeml program from the PAML4 package ${ }^{110}$ with default parameters except for the RateAncestor $=1$ option, to execute the ancestral state reconstruction process. A phylogenetic tree that includes the listed species was extracted from the more extensive tree (See above and Fig. 5) with a conventional trifurcation in its root to designate an unrooted tree (Supplementary Fig. 6). The ancestral coding sequences were evaluated for the LCA of the above-specified primates, as well as for all other intermediate ancestors between human and the LCA. The ancestral nucleotides for each editing site were retrieved from the joint reconstruction multiple sequence alignment of these sequences.

Next, based on their translated amino acids, A-to-G editing sites were classified to (1) synonymous sites where the RNA editing does not alter the translated amino acid, (2) nonsynonymous restorative sites where the amino-acid encoded by the edited human transcript appears at the same position in one or more of the ancestral protein sequences, and (3) nonsynonymous diversifying sites where editing of the human sequence generates a novel amino acid, that does not appear (at the same position) in any of the ancestral protein sequences. A subset of 828 sites were classified in total.

The same methodology was applied to all genes in the RefSeq annotation (see above) regarding all adenosines as putative RNA editing sites in order to assess the frequencies of restorative, diversifying, and synonymous sites relative to their genomic background. Additionally, $10^{6}$ sets of 828 randomly-selected sites were chosen out from the sites in the genomic background with the same distribution over sequence contexts ( 1 bp downstream and upstream), as a control. Randomization-based $p$-values were evaluated using these sets.

Strand-specific RNA-seq validation. To show that T-to-C substitutions detected by our pipeline are mostly due to ADAR-associated A-to-I editing of transcripts expressed from the strand opposing the coding RefSeq strand, we analyzed four additional datasets of strand-specific human RNA-seq samples ${ }^{36}$. One dataset included 18 samples from 6 human tissues (brain, liver, lung, muscle, heart, and kidney) (SRA accession number: SRP058632). The second included 126 retina samples $^{111}$ (all healthy samples from accession number: GSE115828, excluding SRR7461061 which failed our quality checks). The third is composed of 143 fibroblast cell line samples ${ }^{112}$ (accession: GSE113957).

The fourth dataset was created as follows: matched Genomic DNA and Total RNA samples of peripheral blood leukocytes from three adult male humans were obtained from AMSBIO (D1234148 and R1234148-10). Whole exome library was created with the genomic DNA using the Agilent SureSelect XT Human All Exon V6 + UTR protocol. Stranded mRNA library was created with the total RNA using illumina TruSeq Stranded protocol with polyA enrichment. Libraries were sequenced by Macrogen Europe as 150 bp paired end reads using Illumina NovaSeq6000. These newly generated blood sequencing data were deposited to SRA (BioProject ID: PRJNA715360).

These four datasets were processed as described above (Reads Alignment and BAM processing). Total coverage and the number of reads supporting the pipelinedetected A-to-G and T-to-C substitutions were calculated using mpileup, as previously mentioned. Following calculation of per-strand coverage and number of reads supporting the substitution, we applied binomial test (for each strand separately) to test whether the mismatch-harboring reads may be attributed to random sequencing errors (with a rate of $10^{-3}$, corresponding to $q=30$ ). For each mismatch type and strand, $p$-values were adjusted using Benjamini and Hochberg's False Discovery Rate $(F D R=0.05)$ correction. In addition, we have calculated the (per strand) editing index (see above) over the pipeline-detected A-to-G and T-toC substitutions.

Dimensionality reduction analysis. Per-Sample editing levels were calculated for each detected A-to-G site with coverage $\geq 10$ reads, otherwise, the editing level was considered missing. Biological samples that had more the one GTEx sample barcode were not included. Sites or samples with $>80 \%$ missing values were discarded The remaining missing values were then imputed by the mean editing level at this site over all GTEx samples. The kidney samples were removed from the analysis due to the low number of samples $(n=38)$, compared to other tissue types $(n \geq 69)$. t-SNE was performed using the Rtsne package (version 0.15 ; https:// github.com/jkrijthe/Rtsne), with default parameters, except for $\theta=0.25$ for better accuracy. Results were plotted using the ggplot2 package (version 3.2.1).

Editing sites classification by targeting ADAR. We used previously published ADARs overexpression (OE) ${ }^{14}$ (SRA accession: SRP090260; HEK293 cells) and knockout (KO) ${ }^{49}$ (SRA accession: SRP200481; C57BL/6 mice brains) RNA-seq datasets. Alignment of reads to the human or mouse reference genome and quantification of per site total coverage and number of edited reads were performed as described above (Reads Alignment and BAM processing; Recalculating Editing Levels and Editing-Level-Based Filtration; and Coverage calculation in nonhuman samples). As the analysis of editing in mouse was dependent on liftOver 
conversion, 439 sites were excluded due to a non-coherent or a non-A conversion result, leaving 1078 sites. Importantly, none of these sites have shown A-to-G mismatches at an appreciable level in ADAR double-knockout samples (Supplementary Data 13). First, biological replicates were pooled, and Fisher's exact test was applied, comparing the numbers of edited and non-edited reads per site in each targeted ADAR and genetic modification to those in the appropriate WT control. A site was considered as enzyme-specific if its editing level was found to be significantly altered (Benjamini-Hochberg-corrected $p$-value $\leq 0.05$ ) by either the $\mathrm{OE}$ or the KO for one of the ADARs but not for the other and if the editing level was changed in agreement with the nature of the modification (i.e., higher editing levels for $\mathrm{OE}$ and lower for $\mathrm{KO}$ ). When significant $p$-values were obtained for both Adar1 and Adar2 the site was considered as a shared target of both enzymes.

In addition, we estimated the relative influence of Adarl $(I)$ on the editing level at site as follows:

$$
I_{i}=\frac{\log _{2}\left(\frac{\text { WT editing level }+0.001}{\text { Adar1 KO editing level }+0.001}\right)}{\log _{2}\left(\frac{\text { WT editing level }+0.001}{\text { Adar1 KO editing level }+0.001}\right)+\log _{2}\left(\frac{\text { WT editing level }+0.001}{\text { Adar2 KO editing levels }+0.001}\right)}
$$

Negative values of $I_{i}$ were replaced by $I_{i}=0$.

Identification of ECS. To search for complementary sequences, we retrieved a 4lbp-long genomic sequence flanking each of the editing sites (20 bp in each side) and used AB_BLAST (release: 2020-03-17; https://www.advbiocomp.com/ blast.html) to look for a reversely-oriented complementary sequence within 20,001 bp (10k in each side) surrounding the editing site (command: ab-blastn $W=4 Q=14 R=4$-matrix=RNA hspmax =5). Hits of length $\geq 30$ and identity $\geq 70 \%$, residing within the same transcript or up to $5 \mathrm{~kb}$ downstream to it (accounting for long, unannotated UTRs) were considered as putative ECS. As a control, we randomly chose 1517 exonic adenosines that are not known to be edited. For both editing and control sites, minimum free energy $(\Delta G)$ was calculated for the longest BLAST hit (within the transcript or 5000 bp downstream, but regardless of its length and identity) applying the fold program from the RNAStructure package ${ }^{113}$ to the query region and the subject region of the hit, connected with a $100 \mathrm{bp}$ poly(A) linker when needed. Sites for which either the calculated free energy was positive or no hit was found were assigned $\Delta G=0$.

Multi-species RNA secondary structure comparisons. Multi-species dsRNA structures were calculated for regions within 3 genes: ASNS, NEIL1 and SEMA5B. Human ECS were detected as mentioned above with the exception of SEMA5B, where the BLAST-dependent analysis was not sensitive enough to detect the ECS. Instead, a 72 bp-long region that harbors the single editing site in the coding sequence of SEMA5B and found to form a dsRNA structure in-silico was used. Next, nonhuman homologous sequences of the edited region and ECS were retrieved, using the UCSC multiz30way (ASNS and SEMA5B) and multiz100way (NEIL1) tables. RNA secondary structures were predicted, for each gene separately, using the RNAStructure Multilign program with default parameters. Sequences from species with non-editable CDS variants were folded separately from editable ones. Next, to determine the position of mismatches between the homologous sequence we used Clustal Omega (Ver. 1.2.4) ${ }^{108}$ program to generate their multiple sequence alignment.

Proteomic evidence for editing. In order to detect editing events at the protein level we downloaded mass spectrometry data, available at the PRIDE database ${ }^{40}$. We analyzed 311 proteomic and phosphoproteomic samples, (Supplementary Data 4) originated from various human tissues, digested using trypsin, and quantified using label-free quantification (LFQ), tandem mass tag (TMT), or isobaric tag for relative and absolute quantification (iTRAQ).

To prepare a database of all possible peptides, including the ones derived from the 1036 nonsynonymous RNA editing sites in our set, we used RefSeq transcripts and considered all possible combinations of edited and non-edited states at all Ato-G editing sites detected by our pipeline. The translated transcripts were in silico trypsin-digested into peptides, taking into account up to two miss-cleavages per peptide. We considered potential trypsin cleavages losses and gains due to editing but not stop losses. In addition to this set of peptides, we created two additional peptide databases as controls: (i) a proteome where RefSeq transcripts may be "edited" at the 851 nonsynonymous non-A-to-G sites detected by our pipeline (sites we consider as noise). Some of these are likely to be found in the proteome, as they represent genomic variability or misalignment due to genomic duplications. (ii) A proteome where RefSeq transcripts may be "edited" at 1036 randomlychosen genomic positions to create a nonsynonymous change. These random sites were matched to the 1036 detected recoding sites in terms of the edited codon, and (if possible) were chosen within the same exon as the true edited site ( 829 sites). In cases an identical codon was not found in the same exon, we picked a position in the same codon from another exon of the same gene (187 sites) if possible, or in other genes otherwise (20 sites).

The proteomic samples were compared to this set of potential peptides using MaxQuant (MQ) ${ }^{114}$, with default parameters and several modifications, as follows: for phosphoproteome samples we added phosphor-STY modification to the variable modifications list; LFQ samples were analyzed with the parameter lfqmode $=1$ for delayed normalization using MQ LFQ algorithm; for TMT and for iTRAQ samples we chose 11plex-TMT and 8plex-iTRAQ in the isobaric labels parameters section, respectively (with reporter ion MS2). Peptides that may have derived from common contaminants (based on MQ Contaminants Fasta file) were excluded. We then considered only peptides that are distinct to either the nonedited or the edited versions. For each editing site and each sample, we recorded peptides that support the genomic version, the edited version, or both. The same search was applied to the two control databases.

Estimating biological variability of editing levels. To assess the variability of editing across donors, we first summed the total coverage and the number of edited reads over a number of tissues. To avoid variability due to a different set of available tissue samples, we wanted to have the exact same set of tissues for all donors considered. Since not all tissues are available for all donors, we had to choose a subset of donors and a subset of tissues, trying to keep both the number of donors and the number of brain regions sufficiently large to gain statistical power. Focusing on the brain, we studied 65 donors for each of which we pooled the coverage and editing data from five brain regions: caudate, nucleus accumbent, cerebellum, cerebellar hemisphere, and putamen.

Next, we used the following model to separate the stochastic sampling noise and estimate the biological variability across samples. For each editing site, denote $c_{i}$ and $g_{i}$ the total coverage and number of edited reads in sample $i$, respectively. The observed editing level per sample is therefore $e_{i}=g_{i} / c_{i}$. We assume $g_{i}$ to be distributed binomially $g_{i} \sim B\left(c_{i}, p_{i}\right)$, where $p_{i}$ is the underlying editing level per site per sample (i.e., fraction of edited cDNA molecules). This level may be different from sample to sample, and we denote its mean and variance over the population by $p, v$, respectively. The mean observed level is thus

$$
\left\langle e_{i}\right\rangle_{S, B}=\left\langle\frac{g_{i}}{c_{i}}\right\rangle_{S, B}=\left\langle p_{i}\right\rangle_{S}=p
$$

where $\langle\ldots\rangle_{S, B}$ denotes averaging over both samples $(S)$ and the binomial distribution $(B)$, and we have used the relation $\left\langle g_{i}\right\rangle_{B}=p_{i} c_{i}$. The variance of the observed editing level (over both samples and binomial distributions) is

$$
\begin{aligned}
\operatorname{Var}\left(e_{i}\right) & =\left\langle e_{i}^{2}\right\rangle_{S, B}-\left\langle e_{i}\right\rangle_{S, B} 2=\left\langle\frac{\left\langle g_{i}{ }^{2}\right\rangle_{B}}{c_{i}^{2}}\right\rangle_{S}-p^{2} \\
& =\left\langle\frac{1}{c_{i}^{2}}\left(c_{i}^{2} p_{i}^{2}+c_{i} p_{i}\left(1-p_{i}\right)\right)\right\rangle_{S}-p^{2}=\left\langle p_{i}{ }^{2}\right\rangle_{S}-p^{2}+\left\langle\frac{1}{c_{i}} p_{i}\left(1-p_{i}\right)\right\rangle_{S} \\
& =v+\left\langle\frac{1}{c_{i}}\right\rangle_{S}\left\langle p_{i}\left(1-p_{i}\right)\right\rangle_{S}=v\left(1-c^{-1}\right)+c^{-1}\left(p-p^{2}\right)
\end{aligned}
$$

where $c^{-1}=\left\langle\frac{1}{c_{i}}\right\rangle_{S}$ and the penultimate equation assumes that coverage and editing level distributions over samples are uncorrelated. One thus obtains the following for the variance over the population of the underlying editing level:

$$
v=\frac{\operatorname{Var}\left(e_{i}\right)-c^{-1}\left(p-p^{2}\right)}{1-c^{-1}}
$$

For a given set of samples, one may estimate $\mathrm{p}$ and $c^{-1}$ as the mean (over samples) of $e_{i}$ and $\frac{1}{c_{i}}$, respectively, and then apply the above formula to obtain an estimate $v$. Note that this estimate may occasionally turn out negative, due to statistical errors in these estimates. In these cases, our best estimate for the variability in the underlying editing level is zero. Finally, we point out that some of the variability across samples of the underlying editing level may be due to technical rather than biological reasons (sample-to-sample differences in the sequencing protocol that may have an effect on the variance). The above estimate is therefore an over-estimate of the biological variability.

Differentially edited sites in cancer and cardiovascular diseases. To look for differential editing in cancer, RNA-seq samples for tumor samples and matching controls were downloaded from The Cancer Genome Atlas ${ }^{62}$. Following ${ }^{59}$, we considered the nine cancer types for which healthy controls are available. Reads were re-aligned to the genome and editing levels were calculated per sample per site, as described above (Reads Alignment and BAM processing; and Recalculating Editing Levels and Editing-Level-Based Filtration).

For each donor, we excluded sites for which the number of reads aligned in the normal and tumor samples combined was $<40$. Then, we calculated the editing level in the pooled normal samples and the pooled tumor samples, separately, and excluded sites for which both editing levels were $<0.5 \%$ in any of the cancer types. Paired-samples student's t-test was then applied to the remaining sites, for each tissue type, to compare the editing levels per sample between the matched normal and tumor groups, followed by Benjamini-Hochberg multiple testing correction $(\mathrm{FDR}=0.05)$. We further requested an appreciable $>10 \%$ difference in the mean editing level between the groups.

Similarly, we looked at the editing levels observed in the three artery tissue types available in GTEx (aorta, coronaries, and tibial). For each tissue and site, we looked at the 27 diseases and conditions annotated in GTEx. We discarded low-coverage 
donors ( $<20$ reads), and applied Mann-Whitney $U$ test to compare editing level per site between healthy and diseased subjects, provided that both groups included at least 10 subjects. Benjamini-Hochberg FDR correction was applied (FDR $=0.05$ ) per-disease per-tissue, and we further requested an appreciable $>10 \%$ difference in the mean editing level between the groups. None of the sites identified is adjacent $(<2000$ bp distance) to known GWAS loci.

\section{Single-cell differential RNA editing analysis. We used Tabula Muris ${ }^{115}$, a} recently-published comprehensive mouse single-cell dataset to test for variations in editing levels between different cell populations within the same tissue. Smart-seq2 full-length RNA-seq data from 44,494 different cells (SRA accession: SRP131661) were aligned as performed for other nonhuman samples (see above). Total coverage at the editing sites and editing levels were assessed as described above (Recalculating Editing Levels and Editing-Level-Based Filtration) for the classification by targeting ADAR. Next, per-cell total coverage and editing values were summarized across all cells in each cell population based on the previouslypublished annotations ${ }^{115}$. As for lung cells, a revised and more accurate annotation was used $^{116}$ (Supplementary Data 6). Then, sites that did not exhibit an editing level $\geq 10 \%$ with minimal coverage of 5 reads in at least one cell population were excluded, and for each site, all possible pairs of cell populations within the same tissue were compared to detect significant differences in editing levels. A total of 14,503 pairs were evaluated in both the cell-population and the single-cell levels. At the cell-population level, the total numbers of edited and non-edited reads for each pair were compared using Fisher's exact test. To assess differential editing levels using single-cell data, per-cell editing levels were calculated for all sites that were considered for the analysis, and compared between different cell populations within the same tissue using Mann-Whitney $U$ test. $P$ values from both comparisons were adjusted using the Benjamini-Yekutieli method. Comparisons with adjusted $p \leq 0.05$ are shown in Supplementary Data 7 .

Reporting summary. Further information on research design is available in the Nature Research Reporting Summary linked to this article.

\section{Data availability}

The data supporting the findings of this study are available from the corresponding authors upon reasonable request. Human data used in the paper is available upon $\mathrm{dbGaP}$ approval (GTEx: phs000424.v8.p2; TCGA: phs000178.v11.p8). Newly generated nextgeneration sequencing data reported in this study have been deposited in the SRA database, BioProject ID: PRJNA715360. Non-human RNA-seq samples analyzed are available from SRA (see detailed in Supplementary Data 13). Source data are provided with this paper.

\section{Code availability}

The scripts used to produce the data are available at GitHub (https://github.com/ a2iediting/deNovo-Detect) ${ }^{117}$.

Received: 28 April 2021; Accepted: 27 January 2022; Published online: 04 March 2022

\section{References}

1. Walkley, C. R. \& Li, J. B. Rewriting the transcriptome: Adenosine-to-inosine RNA editing by ADARs. Genome Biol. 18, 205 (2017).

2. Nishikura, K. A-to-I editing of coding and non-coding RNAs by ADARs. Nat. Rev. Mol. Cell Biol. 17, 83-96 (2016).

3. Eisenberg, E. \& Levanon, E. Y. A-to-I RNA editing-immune protector and transcriptome diversifier. Nat. Rev. Genet. 19, 473-490 (2018).

4. Bass, B. L. RNA editing by adenosine deaminases that act on RNA. Annu. Rev. Biochem. 71, 817-846 (2002).

5. Bazak, L. et al. A-to-I RNA editing occurs at over a hundred million genomic sites, located in a majority of human genes. Genome Res. 24, 365-376 (2014).

6. Basilio, C., Wahba, A. J., Lengyel, P., Speyer, J. F. \& Ochoa, S. Synthetic polynucleotides and the amino acid code. Proc. Natl Acad. Sci. USA 48 613-616 (1962).

7. Higuchi, M. et al. Point mutation in an AMPA receptor gene recues lethality in mice deficient in the RNA-editing enzyme ADAR2. Nature 406, 1998-2001 (2000).

8. Chen, L. et al. Recoding RNA editing of AZIN1 predisposes to hepatocellular carcinoma. Nat. Med. 19, 209-216 (2013).

9. Yeo, J., Goodman, R. A., Schirle, N. T., David, S. S. \& Beal, P. A. RNA editing changes the lesion specificity for the DNA repair enzyme NEIL1. Proc. Natl Acad. Sci. USA 107, 20715-20719 (2010).

10. Jain, M. et al. RNA editing of Filamin A pre-mRNA regulates vascular contraction and diastolic blood pressure. EMBO J. 37, e94813 (2018).
11. Ramaswami, G. \& Li, J. B. RADAR: A rigorously annotated database of A-to-I RNA editing. Nucleic Acids Res. 42, D109-D113 (2014)

12. Picardi, E., D’Erchia, A. M., Lo Giudice, C. \& Pesole, G. REDIportal: A comprehensive database of A-to-I RNA editing events in humans. Nucleic Acids Res. 45, D750-D757 (2017)

13. Mangul, S. et al. ROP: dumpster diving in RNA-sequencing to find the source of 1 trillion reads across diverse adult human tissues. Genome Biol. 19, 36 (2018).

14. Tan, M. H. et al. Dynamic landscape and regulation of RNA editing in mammals. Nature 550, 249-254 (2017).

15. Park, E., Williams, B., Wold, B. J. \& Mortazavi, A. RNA editing in the human ENCODE RNA-seq data. Genome Res. 22, 1626-1633 (2012).

16. Porath, H. T., Carmi, S. \& Levanon, E. Y. A genome-wide map of hyper-edited RNA reveals numerous new sites. Nat. Commun. 5, 4726 (2014)

17. Zhang, Q. \& Xiao, X. Genome sequence-independent identification of RNA editing sites. Nat. Methods 12, 347-350 (2015).

18. Licht, $\mathrm{K}$. et al. A high resolution A-to-I editing map in the mouse identifies editing events controlled by pre-mRNA splicing. Genome Res. 29, 1453-1463 (2019).

19. Carmi, S. et al. Sequencing an Ashkenazi reference panel supports populationtargeted personal genomics and illuminates Jewish and European origins. Nat. Commun. 5, 4835 (2014).

20. Ramaswami, G. \& Li, J. B. Identification of human RNA editing sites: A historical perspective. Methods 107, 42-47 (2016).

21. Kleinman, C. L. \& Majewski, J. Comment on 'Widespread RNA and DNA sequence differences in the human transcriptome'1. Science 335, 1302 (2012). author reply 1302

22. Lin, W., Piskol, R., Tan, M. H. \& Li, J. B. Comment on "Widespread RNA and DNA sequence differences in the human transcriptome". Science 335, 1302-1302 (2012)

23. Pickrell, J. K., Gilad, Y. \& Pritchard, J. K. Comment on Widespread RNA and DNA sequence differences in the human transcriptome". Science 335, 1302-1302 (2012).

24. Telenti, A. et al. Deep sequencing of 10,000 human genomes. Proc. Natl Acad. Sci. USA 113, 11901-11906 (2016).

25. Abel, H. J. et al. Mapping and characterization of structural variation in 17,795 human genomes. Nature 583, 83-89 (2020).

26. Zaranek, A. W., Levanon, E. Y., Zecharia, T., Clegg, T. \& Church, G. M. A survey of genomic traces reveals a common sequencing error, RNA editing, and DNA editing. PLoS Genet. 6, e1000954 (2010).

27. Ramaswami, G. et al. Identifying RNA editing sites using RNA sequencing data alone. Nat. Methods 10, 128-132 (2013).

28. Ramaswami, G. et al. Accurate identification of human Alu and non-Alu RNA editing sites. Nat. Methods 9, 579-581 (2012).

29. Mele, M. et al. The human transcriptome across tissues and individuals. Science 348, 660-665 (2015).

30. Zhang, F., Lu, Y., Yan, S., Xing, Q. \& Tian, W. SPRINT: An SNP-free toolkit for identifying RNA editing sites. Bioinformatics 33, 3538-3548 (2017).

31. Giudice, C. L., Tangaro, M. A., Pesole, G. \& Picardi, E. Investigating RNA editing in deep transcriptome datasets with REDItools and REDIportal. Nat. Protoc. 15, 1098-1131 (2020)

32. John, D., Weirick, T., Dimmeler, S. \& Uchida, S. RNAEditor: Easy detection of RNA editing events and the introduction of editing islands. Brief. Bioinform. 18, 993-1001 (2017).

33. Picardi, E. et al. Profiling RNA editing in human tissues: towards the inosinome Atlas. Sci. Rep. 5, 14941 (2015).

34. Lonsdale, J. et al. The Genotype-Tissue Expression (GTEx) project. Nat. Genet. 45, 580-585 (2013).

35. Eggington, J. M., Greene, T. \& Bass, B. L. Predicting sites of ADAR editing in double-stranded RNA. Nat. Commun. 2, 319 (2011)

36. D'Erchia, A. M. et al. Tissue-specific mtDNA abundance from exome data and its correlation with mitochondrial transcription, mass, and respiratory activity. Mitochondrion 20, 13-21 (2015)

37. Rosenberg, B. R., Hamilton, C. E., Mwangi, M. M., Dewell, S. \& Papavasiliou, F. N. Transcriptome-wide sequencing reveals numerous APOBEC1 mRNA editing targets in transcript $3^{\prime}$ UTRs. Nat. Struct. Mol. Biol. 18, 230 (2011).

38. Powell, L. M. et al. A novel form of tissue-specific RNA processing produces apolipoprotein-B48 in intestine. Cell 50, 831-840 (1987).

39. Polson, A. G., Crain, P. F., Pomerantz, S. C., McCloskey, J. A. \& Bass, B. L. The mechanism of adenosine to inosine conversion by the double-stranded RNAunwinding/modifying activity: A high-performance liquid chromatographymass spectrometry analysis. Biochemistry 30, 11507-11514 (1991).

40. Perez-Riverol, Y. et al. The PRIDE database and related tools and resources in 2019: Improving support for quantification data. Nucleic Acids Res. 47, D442-D450 (2019).

41. Michalski, A. et al. Ultra high resolution linear ion trap Orbitrap mass spectrometer (Orbitrap Elite) facilitates top down LC MS/MS and versatile peptide fragmentation modes. Mol. Cell. Proteomics 11, O111.013698 (2012). 
42. Pinto, Y., Cohen, H. Y. \& Levanon, E. Y. Mammalian conserved ADAR targets comprise only a small fragment of the human editosome. Genome Biol. 15, R5 (2014).

43. Levanon, E. Y. et al. Evolutionarily conserved human targets of adenosine to inosine RNA editing. Nucleic Acids Res. 33, 1162-1168 (2005).

44. Roth, S. H., Levanon, E. Y. \& Eisenberg, E. Genome-wide quantification of ADAR adenosine-to-inosine RNA editing activity. Nat. Methods 16, 1131-1138 (2019).

45. Silvestris, D. A. et al. Dynamic inosinome profiles reveal novel patient stratification and gender-specific differences in glioblastoma. Genome Biol. 20, 33 (2019).

46. Gal-Mark, N. et al. Abnormalities in A-to-I RNA editing patterns in CNS injuries correlate with dynamic changes in cell type composition. Sci. Rep. 7, 43421 (2017)

47. Oakes, E., Anderson, A., Cohen-Gadol, A. \& Hundley, H. A. Adenosine deaminase that acts on RNA 3 (ADAR3) binding to glutamate receptor subunit B Pre-mRNA inhibits RNA editing in glioblastoma. J. Biol. Chem. 292, 4326-4335 (2017).

48. Hodge, R. D. et al. Conserved cell types with divergent features in human versus mouse cortex. Nature 573, 61-68 (2019).

49. Chalk, A. M., Taylor, S., Heraud-Farlow, J. E. \& Walkley, C. R. The majority of A-to-I RNA editing is not required for mammalian homeostasis. Genome Biol. 20, 268 (2019).

50. Greenberger, S. et al. Consistent levels of A-to-I RNA editing across individuals in coding sequences and non-conserved Alu repeats. BMC Genomics 11, 608 (2010).

51. Morse, D. P., Aruscavage, P. J. \& Bass, B. L. RNA hairpins in noncoding regions of human brain and Caenorhabditis elegans mRNA are edited by adenosine deaminases that act on RNA. Proc. Natl Acad. Sci. USA 99, 7906-7911 (2002).

52. Levanon, E. Y. et al. Systematic identification of abundant A-to-I editing sites in the human transcriptome. Nat. Biotechnol. 22, 1001-5 (2004).

53. Blow, M., Futreal, P. A., Wooster, R. \& Stratton, M. R. A survey of RNA editing in human brain. Genome Res. 14, 2379-2387 (2004).

54. Kim, D. D. Y. et al. Widespread RNA editing of embedded alu elements in the human transcriptome. Genome Res. 14, 1719-1725 (2004).

55. Athanasiadis, A., Rich, A. \& Maas, S. Widespread A-to-I RNA editing of Alucontaining mRNAs in the human transcriptome. PLoS Biol. 2, e391 (2004).

56. Miura, P., Shenker, S., Andreu-Agullo, C., Westholm, J. O. \& Lai, E. C. Widespread and extensive lengthening of 39 UTRs in the mammalian brain. Genome Res. 23, 812-825 (2013).

57. Gallo, A., Vukic, D., Michalík, D., O’Connell, M. A. \& Keegan, L. P. ADAR RNA editing in human disease; more to it than meets the I. Hum. Genet. 136, 1265-1278 (2017).

58. Hwang, T. et al. Dynamic regulation of RNA editing in human brain development and disease. Nat. Neurosci. 19, 1093-1099 (2016).

59. Paz-Yaacov, N. et al. Elevated RNA editing activity is a major contributor to transcriptomic diversity in tumors. Cell Rep. 13, 267-276 (2015).

60. Han, L. et al. The genomic landscape and clinical relevance of A-to-I RNA editing in human cancers. Cancer Cell 28, 515-28 (2015).

61. Fumagalli, D. et al. Principles governing A-to-I RNA editing in the breast cancer transcriptome article principles governing A-to-I RNA editing in the breast cancer transcriptome. Cell Rep. 13, 277-289 (2015).

62. The Cancer Genome Atlas Research Network. The Cancer Genome Atlas PanCancer analysis project. Nat. Genet. 45, 1113-1120 (2013).

63. Jiang, Y. et al. The sheep genome illuminates biology of the rumen and lipid metabolism. Science 344, 1168-1173 (2014).

64. Yang, X.-Z. et al. Selectively constrained RNA editing regulation crosstalks with piRNA biogenesis in primates. Mol. Biol. Evol. 32, 3143-3157 (2015).

65. Liao, X. et al. Sequence, structural, and expression divergence of duplicate genes in the bovine genome. PLoS One 9, e102868 (2014).

66. Correia, C. N. et al. RNA sequencing (RNA-Seq) reveals extremely low levels of reticulocyte-derived globin gene transcripts in peripheral blood from horses (Equus caballus) and cattle (Bos taurus). Front. Genet. 9, 278 (2018).

67. Peng, $X$. et al. Tissue-specific transcriptome sequencing analysis expands the non-human primate reference transcriptome resource (NHPRTR). Nucleic Acids Res. 43, D737-D742 (2015).

68. Cardoso-Moreira, M. et al. Gene expression across mammalian organ development. Nature 571, 505-509 (2019).

69. Riemondy, K. A. et al. Dynamic temperature-sensitive A-to-I RNA editing in the brain of a heterothermic mammal during hibernation. RNA 24, 1481-1495 (2018).

70. Söllner, J. F. et al. An RNA-Seq atlas of gene expression in mouse and rat normal tissues. Sci. Data 4, 170185 (2017).

71. Zhang, Y. et al. Genome-wide profiling of RNA editing sites in sheep. J. Anim. Sci. Biotechnol. 10, 31 (2019).

72. Ruiz-Orera, J. et al. Origins of De Novo Genes in Human and Chimpanzee. PLoS Genet. 11, e1005721 (2015).
73. Chen, J.-Y. et al. RNA editome in rhesus macaque shaped by purifying selection. PLoS Genet. 10, e1004274 (2014).

74. Zhang, Y. et al. Genome-wide identification of RNA editing in seven porcine tissues by matched DNA and RNA high-throughput sequencing. J. Anim. Sci. Biotechnol. 10, 24 (2019).

75. Tang, Z. et al. Comprehensive analysis of long non-coding RNAs highlights their spatio-temporal expression patterns and evolutional conservation in Sus scrofa. Sci. Rep. 7, 1-12 (2017).

76. $\mathrm{Hu}, \mathrm{J}$. et al. Whole blood transcriptome sequencing reveals gene expression differences between Dapulian and Landrace piglets. Biomed. Res. Int. 2016, 7907980 (2016)

77. Xie, C. et al. Hominoid-specific de novo protein-coding genes originating from long non-coding RNAs. PLoS Genet. 8, e1002942 (2012).

78. Yu, Y. et al. A rat RNA-Seq transcriptomic BodyMap across 11 organs and 4 developmental stages. Nat. Commun. 5, 3230 (2014)

79. Bowyer, J. F. et al. Evaluating the stability of RNA-Seq transcriptome profiles and drug-induced immune-related expression changes in whole blood. PLoS One 10, e0133315 (2015).

80. Funkhouser, S. A. et al. Evidence for transcriptome-wide RNA editing among Sus scrofa PRE-1 SINE elements. BMC Genomics 18, 360 (2017).

81. Li, B. et al. A comprehensive mouse transcriptomic BodyMap across 17 tissues by RNA-seq. Sci. Rep. 7, 1-10 (2017).

82. Dillman, A. A. et al. MRNA expression, splicing and editing in the embryonic and adult mouse cerebral cortex. Nat. Neurosci. 16, 499-506 (2013).

83. Ropka-Molik, K. et al. Transcriptome profiling of Arabian horse blood during training regimens. BMC Genet. 18, 31 (2017).

84. Sousa, A. M. M. et al. Molecular and cellular reorganization of neural circuits in the human lineage. Science 358, 1027-1032 (2017).

85. $\mathrm{Xu}, \mathrm{C}$. et al. Human-specific features of spatial gene expression and regulation in eight brain regions. Genome Res. 28, 1097-1110 (2018).

86. Yang, Y. et al. Comparative analysis of DNA methylome and transcriptome of skeletal muscle in lean-, obese-, and mini-type pigs. Sci. Rep. 7, 1-14 (2017)

87. Mure, L. S. et al. Diurnal transcriptome atlas of a primate across major neural and peripheral tissues. Science 359, eaao0318 (2018).

88. Merkin, J., Russell, C., Chen, P. \& Burge, C. B. Evolutionary dynamics of gene and isoform regulation in Mammalian tissues. Science 338, 1593-9 (2012).

89. Choi, J. et al. Haemopedia RNA-seq: A database of gene expression during haematopoiesis in mice and humans. Nucleic Acids Res. 47, D780-D785 (2019).

90. Yue, F. et al. A comparative encyclopedia of DNA elements in the mouse genome. Nature 515, 355-364 (2014)

91. Yim, H. S. et al. Minke whale genome and aquatic adaptation in cetaceans. Nat. Genet. 46, 88-92 (2014).

92. Brawand, D. et al. The evolution of gene expression levels in mammalian organs. Nature 478, 343-348 (2011).

93. Morey, J. S. et al. RNA-Seq analysis of seasonal and individual variation in blood transcriptomes of healthy managed bottlenose dolphins. BMC Genomics 17, 720 (2016).

94. Xu, G. \& Zhang, J. Human coding RNA editing is generally nonadaptive. Proc. Natl Acad. Sci. USA 111, 3769-74 (2014).

95. Jiang, D. \& Zhang, J. The preponderance of nonsynonymous A-to-I RNA editing in coleoids is nonadaptive. Nat. Commun. 10, 5411 (2019).

96. Daniel, C., Silberberg, G., Behm, M. \& Ohman, M. Alu elements shape the primate transcriptome by cis-regulation of RNA editing. Genome Biol. 15, R28 (2014).

97. Picardi, E., Horner, D. S. \& Pesole, G. Single-cell transcriptomics reveals specific RNA editing signatures in the human brain. RNA 23, 860-865 (2017)

98. Ansell, B. R. E. et al. A survey of RNA editing at single-cell resolution links interneurons to schizophrenia and autism. RNA. 27, 1482-1496 (2021).

99. Dobin, A. et al. STAR: Ultrafast universal RNA-seq aligner. Bioinformatics 29, 15-21 (2013).

100. Kent, W. J. et al. The human genome browser at UCSC. Genome Res. 12, 996-1006 (2002).

101. Jun, G., Wing, M. K., Abecasis, G. R. \& Kang, H. M. An efficient and scalable analysis framework for variant extraction and refinement from populationscale DNA sequence data. Genome Res. 25, 918-925 (2015).

102. Auton, A. et al. A global reference for human genetic variation. Nature 526, 68-74 (2015)

103. Bazak, L., Levanon, E. Y. \& Eisenberg, E. Genome-wide analysis of Alu editability. Nucleic Acids Res. 42, 6876-84 (2014).

104. Kent, W. J. BLAT-The BLAST-like alignment tool. Genome Res. 12, 656-664 (2002).

105. Wang, K., Li, M. \& Hakonarson, H. ANNOVAR: Functional annotation of genetic variants from high-throughput sequencing data. Nucleic Acids Res. 38, e164 (2010)

106. Quinlan, A. R. \& Hall, I. M. BEDTools: A flexible suite of utilities for comparing genomic features. Bioinformatics 26, 841-842 (2010). 
107. Letunic, I. \& Bork, P. Interactive Tree of Life (iTOL) v4: Recent updates and new developments. Nucleic Acids Res. 47, W256-W259 (2019).

108. Sievers, F. et al. Fast, scalable generation of high-quality protein multiple sequence alignments using Clustal Omega. Mol. Syst. Biol. 7, 539 (2011).

109. Suyama, M., Torrents, D. \& Bork, P. PAL2NAL: Robust conversion of protein sequence alignments into the corresponding codon alignments. Nucleic Acids Res. 34, W609-12 (2006).

110. Yang, Z. PAML 4: Phylogenetic analysis by maximum likelihood. Mol Biol. Evol. https://doi.org/10.1093/molbev/msm088 (2007).

111. Brooks, M. J. et al. Improved retinal organoid differentiation by modulating signaling pathways revealed by comparative transcriptome analyses with development in vivo. Stem Cell Rep. 13, 891-905 (2019).

112. Fleischer, J. G. et al. Predicting age from the transcriptome of human dermal fibroblasts. Genome Biol. 19, 221 (2018).

113. Reuter, J. S. \& Mathews, D. H. RNAstructure: Software for RNA secondary structure prediction and analysis. BMC Bioinform. 11, 129 (2010).

114. Cox, J. \& Mann, M. MaxQuant enables high peptide identification rates, individualized p.p.b.-range mass accuracies, and proteome-wide protein quantification. Nat. Biotechnol. 26, 1367-1372 (2008).

115. Schaum, N. et al. Single-cell transcriptomics of 20 mouse organs creates a Tabula Muris. Nature 562, 367-372 (2018).

116. Travaglini, K. J. et al. A molecular cell atlas of the human lung from single-cell RNA sequencing. Nature 587, 619-625 (2020).

117. Gabay, O. et al. Landscape of adenosine-to-inosine RNA recoding across human tissues. Github https://doi.org/10.5281/zenodo.5787365 (2021)

118. Van Der Maaten, L. \& Hinton, G. Visualizing data using t-SNE. J. Mach. Learn. Res. 9, 2579-2605 (2008).

\section{Acknowledgements}

We thank the GTEx consortium for making their RNA sequencing data publicly available. We thank Ariel Feiglin and Ilana Buchumenski for their help. This research has been supported by the International Collaboration Grant from the Jacki and Bruce Barron Cancer Research Scholars' Program, a partnership of the Israel Cancer Research Fund and City of Hope, as supported by The Harvey L. Miller Family Foundation [205467 to E.Y.L], and the Israel Science Foundation (grant numbers 1945/18 to E.E and 2039/20, 231/21 to E.Y.L.).

\section{Author contributions}

O.G. and E.E. performed most of the bioinformatics data analyses and wrote the software. Y.S. performed the proteomics analysis, E.K. the ADAR assignment, U.B.Z. the
RNA structure analysis, T.D.M. analyzed the clinical data, N.B. worked with the TCGA data, R.C.F. performed t-SNE and S.H.R. has contributed to specific data analyses. A.A.S carries out the sequencing tasks. S.H.R. and Z.T. curated the software. E.Y.L., O.G., and E.E. conceived the study, designed the analyses, and wrote the paper. All authors read and approved the final manuscript.

\section{Competing interests}

The authors declare competing financial interests. E.E. is a consultant to Korro Bio, a company that develops RNA editors. E.Y.L was a consultant to ADARx. The remaining authors declare no competing interests.

\section{Additional information}

Supplementary information The online version contains supplementary material available at https://doi.org/10.1038/s41467-022-28841-4.

Correspondence and requests for materials should be addressed to Erez Y. Levanon or Eli Eisenberg.

Peer review information Nature Communications thanks Rui Zhang, Graziano Pesole, and Meng How Tan for their contribution to the peer review of this work.

Reprints and permission information is available at http://www.nature.com/reprints

Publisher's note Springer Nature remains neutral with regard to jurisdictional claims in published maps and institutional affiliations.
Open Access This article is licensed under a Creative Commons Attribution 4.0 International License, which permits use, sharing, adaptation, distribution and reproduction in any medium or format, as long as you give appropriate credit to the original author(s) and the source, provide a link to the Creative Commons license, and indicate if changes were made. The images or other third party material in this article are included in the article's Creative Commons license, unless indicated otherwise in a credit line to the material. If material is not included in the article's Creative Commons license and your intended use is not permitted by statutory regulation or exceeds the permitted use, you will need to obtain permission directly from the copyright holder. To view a copy of this license, visit http://creativecommons.org/ licenses/by/4.0/.

(C) The Author(s) 2022 